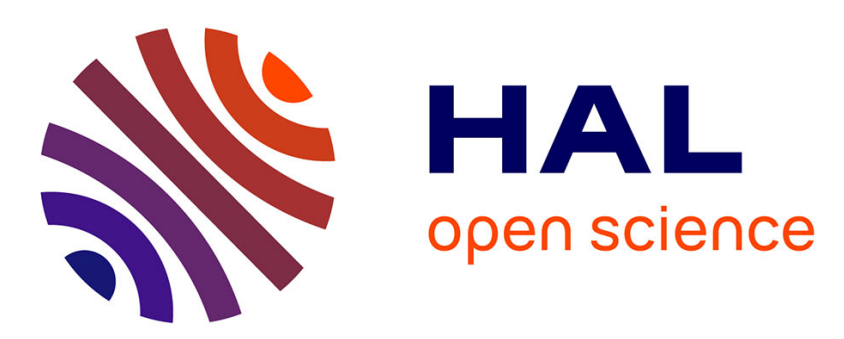

\title{
Handling large model uncertainty in adaptive feedback noise attenuation by overparametrization
}

\author{
Gabriel Buche, Bernard Vau, Ioan Doré Landau, Raul Melendez
}

\section{To cite this version:}

Gabriel Buche, Bernard Vau, Ioan Doré Landau, Raul Melendez. Handling large model uncertainty in adaptive feedback noise attenuation by overparametrization. Journal of Sound and Vibration, 2021, 509 (September), pp.116242. 10.1016/j.jsv.2021.116242 . hal-02959414v2

\section{HAL Id: hal-02959414 \\ https://hal.science/hal-02959414v2}

Submitted on 1 May 2021

HAL is a multi-disciplinary open access archive for the deposit and dissemination of scientific research documents, whether they are published or not. The documents may come from teaching and research institutions in France or abroad, or from public or private research centers.
L'archive ouverte pluridisciplinaire HAL, est destinée au dépôt et à la diffusion de documents scientifiques de niveau recherche, publiés ou non, émanant des établissements d'enseignement et de recherche français ou étrangers, des laboratoires publics ou privés. 


\title{
Handling large model uncertainty in adaptive feedback noise attenuation by overparametrization
}

\author{
Gabriel Buche ${ }^{\mathrm{a}}$, Bernard Vau ${ }^{\mathrm{b}}$, Ioan Doré Landau' ${ }^{1 \mathrm{a}}$, Raul Melendez $^{\mathrm{a}}$ \\ ${ }^{a}$ Univ. Grenoble Alpes, CNRS, Grenoble INP, GIPSA-lab, 38000 Grenoble, France \\ ${ }^{b}$ Ixblue, 94380 Bonneuil-sur-Marne, France
}

\begin{abstract}
Adaptive feedback noise attenuation is a very efficient way of strongly attenuating multiple tonal and narrow band disturbances with unknown and time varying characteristics. These adaptive schemes implement the internal model principle (IMP) for canceling disturbances combined with the Youla Kucera (YK) parametrization which allows to directly tune the disturbance compensation filter without explicit identification of the disturbance model. Efficient use of these schemes requires a good knowledge of the compensatory path model, which can be obtained by experimental system identification. However, there are potential applications where the characteristics of the compensatory path may change significantly during operation and this may lead to the instability of the system. The paper addresses the problem of handling large plant model uncertainties by overparametrization of the adaptive disturbance compensation filter. A methodology for designing adaptive feedback noise cancelers in the presence of large model uncertainties is proposed. In addition to the overparamtrization, a specific design of the linear feedback controller has to be done in order to satisfy a frequency condition in the range of variations of the frequencies characteristics of the compensatory path model. Experimental validation of the design is done on a relevant active noise control bench test.
\end{abstract}

Keywords: active noise control, adaptive feedback compensation, Youla-Kučera parametrization

\section{Introduction}

For the rejection of multiple narrow band noise disturbances with unknown and time-varying characteristics adaptive feedback solutions have shown to be very efficient. Using such a solution, the need of an extra measurement of an image of the disturbance is removed as well as the presence of the undesirable positive internal feedback occurring in most of the adaptive feedforward attenuation schemes.

The essence of the adaptive feedback approach is to use the Internal Model Principle (IMP) [1], which requires, for asymptotic rejection of the disturbance, the inclusion of the disturbance model into the controller, combined with a Youla-Kucera (YK) parametrization of the controller allowing directly to adaptively tune the parameters of the controllers (no need for the explicit identification of the disturbance model).

${ }^{1}$ Corresponding author: Ioan Doré Landau (ioan-dore.landau@ gipsa-lab.grenoble-inp.fr)

Preprint submitted to Journal of Sound and Vibration

March 25, 2021 
The basic diagram of such a system is shown in Fig. 1. In addition to a central polynomial controller (Ro,So) there is a $Q$ filter (FIR) termed the Youla-Kucera filter. This filter has as input the signal $w(t)$ which can be viewed as the output of a disturbance observer [2]

The first use of this approach in active noise attenuation is presented in [3]. A more recent

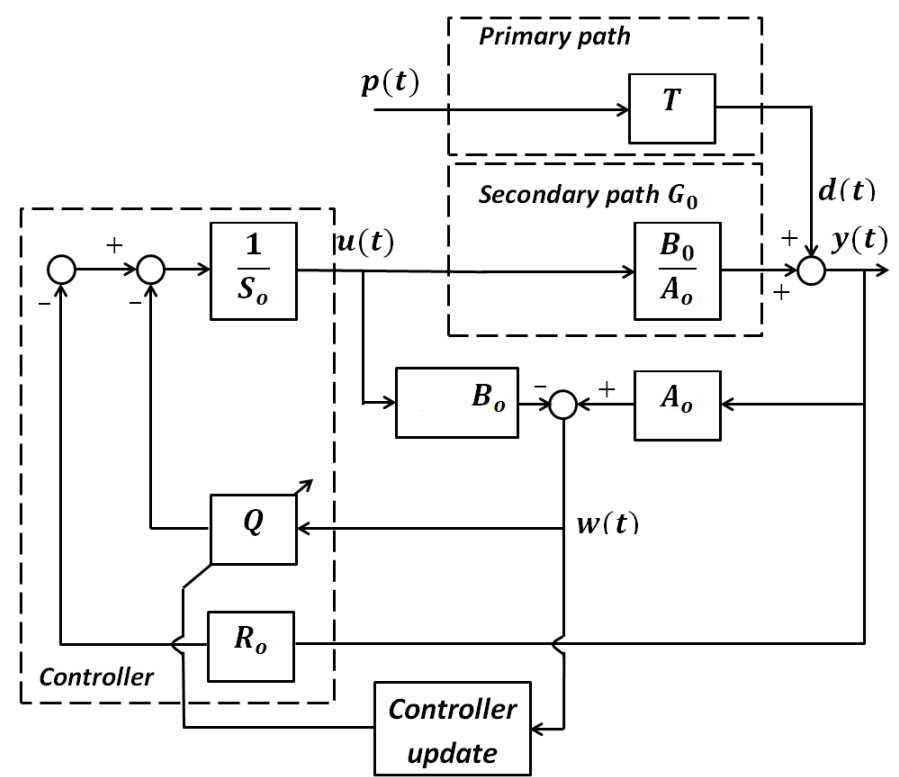

Figure 1: Youla-Kucera direct adaptive feedback regulation scheme.

application is [4]. This approach has been extensively used in active vibration control [2] and has been the subject of an international benchmark reported in a special issue of European Journal of Control [5].

In this context, it is assumed that the models of narrow band noise disturbances are unknown but it is assumed that the compensatory (secondary) path model is known and almost constant. Excellent models of the compensatory path in active noise attenuation can be obtained by experimental system identification (very small differences between simulations and real experiments). See for example [6, 7]. Changes of the physical configurations and of various operational conditions lead to models of the same structure but with different orders and parameters. There are potential applications in active noise attenuation where large variations of the compensatory path model may occur and this will lead in most of the cases to instability of the system. So one of the crucial issues is how to take into account plant model variations.

There are several approaches discussed in the literature for handling model variations. A first approach considers the injection of an external excitation signal allowing to identify in operation the model of the system. However, the optimal choice of the type of signals, of the level and the instants of application of these signals are quite complicated problems. A trade-off has to be found between performance degradation due to model errors and injected signals. This approach is too problem dependent. A second approach considers the performance oriented switching between a collection of previously identified models [8]. It has an interesting potential for large and rapid 
variations of the models. However, fast switching requires to use a limited set of models (see [9], chapter 13) and should be completed with a further adaptation of the model in order to achieve the best performance. Another approach [10] [11],[12],[13] proposes to use an internal error and to identify a model where the place of the controller and of the plant is reversed. This approach has been used in "feedforward compensation" schemes.

The idea of using overparametrization for handling model uncertainties in adaptive Youla-Kucera feedback structures appeared probably for the first time in [14] where an adaptive Youla-Kucera feedback scheme has been used for rejection of an exponential type disturbance acting on a bio reactor characterized by a first order model. It was suggested that overparametrization of the adaptive $\mathrm{Q}$ filter is a possibility for handling not only the disturbance but also the uncertainties on the plant model. In [15] it is argued that overparametrizing the Q filter, enhances the robustness of the control system and that it should be possible to account for changes in the plant models. In [16] an approach for rejecting unknown disturbances acting on an uncertain system using the dual Youla-Kucera parameterization [17] has been proposed. However, no stability analysis is provided for the full scheme. In [18] the analysis of a scheme with an extended adaptive Q filter for the case of a first order system is provided. However these results cannot be extended for systems of higher order like in active noise control where the orders of the plant models can be very high (often over 25).

The analysis of the overparametrization approach has been investigated in [19]. One of the basic finding is that it is not enough to oveparametrize the adaptive filter in order to guarantee the stability of the system. The system with overparametrized Q filter should do simultaneously two tasks: verify the internal model principle while guaranteeing the stability of the system. Even assuming that one knows the uncertainty, it results that there is a frequency condition for stability. In other terms, in addition to using overparametrization, the controller has to be designed such that a frequency condition is satisfied for all the models in the set of possible models.

Furthermore, as a consequence of using overparametrization, the argument of excitation richness of the disturbance can no longer be used for parameter convergence and stability analysis of the adaptive scheme. A specific analysis has to be done (this aspect has not been discussed in [19]). Since one uses overparametrization, the solution of the internal model principle is no more unique and different solutions may result for various initial conditions. However for stability reasons, the domain of possible values of the parameters should be bounded. Therefore the standard parameter adaptation algorithms have to be completed with a projection procedure to maintain the parameters within a certain domain of variations. For the case of unstructured uncertainties, this problem has been discussed in [15]. In the present paper, such a procedure is proposed for the case of structured uncertainties and a stability analysis of the full scheme is provided.

A fundamental message which the paper tries to deliver is the following: A simple overparametrization of the adaptive $Q$ filter is not enough for handling large plant uncertainties. An appropriate design of the linear controller has to be done and the basic adaptation algorithms have to be completed with a projection procedure. The paper also shows that this procedure for handling large model uncertainties can be effectively implemented in adaptive feedback noise attenuation of multiple unknown narrow band disturbances and an experimental evaluation on a relevant test bench is provided.

The paper is organized as follows: In Section 2, the experimental setup will be described. 
The basic equations describing the system will be given in Section 3 . Section 4 will present the methodology of designing the full system in order to handle plant model uncertainties. The experimental results obtained on the test bench are summarized in Section 6. Concluding remarks are presented in Section 7 .

\section{Experimental Setup}

The test bench, used in the paper, allows to test active noise control in pipes for various physical configurations. A detailed scheme of the test bench with the control loop is shown in Fig. 2 and the views of the two implementations which will be considered subsequently are shown in Fig. 3 . The actual dimensions of the two implementations are given in Fig. 4

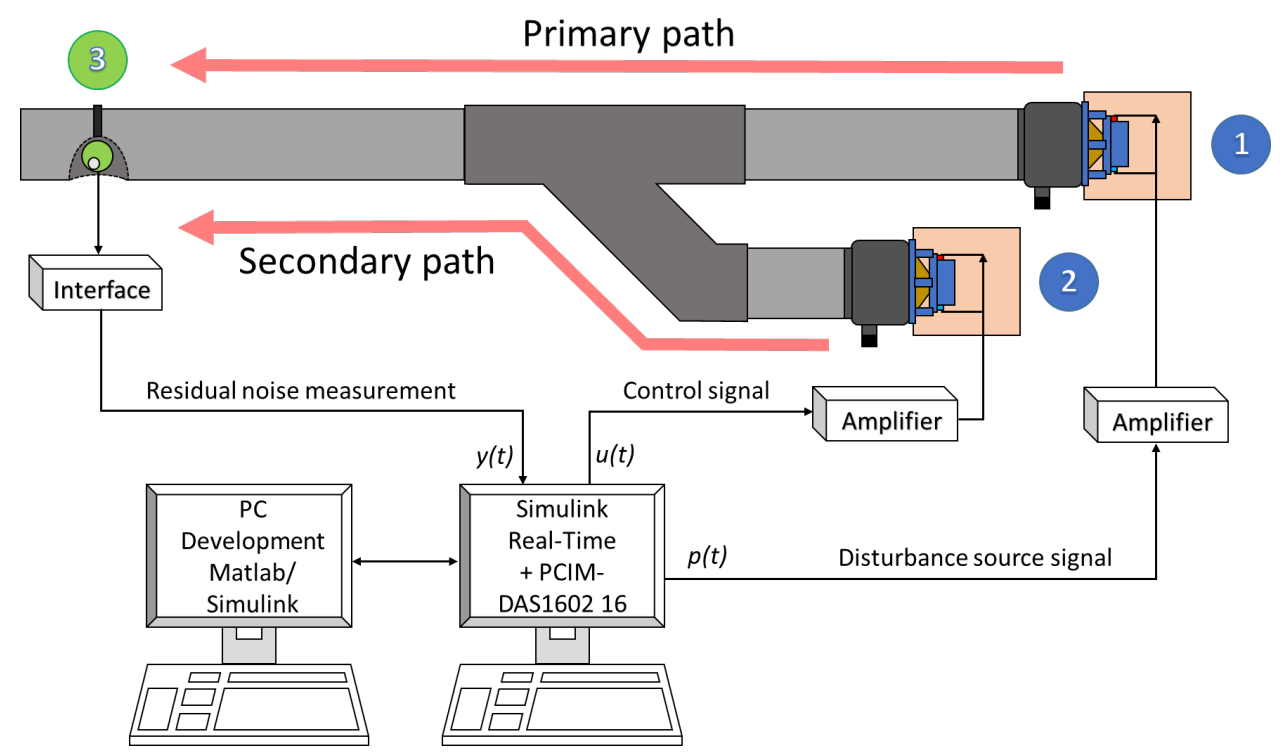

Figure 2: Duct active noise control test bench diagram.

In Fig. 2, the speaker used as the source of disturbances is labelled as 1, while the control speaker is marked as 2. At pipe's open end, the microphone that measures the system's output (residual noise) is denoted as 3 . The control signal is denoted $u(t)$, the residual noise is denoted $y(t)$. The transfer function between the disturbance's speaker and the microphone $(1 \rightarrow 3)$ is called Primary Path, while the transfer function between the control speaker and the microphone $(2 \rightarrow 3)$ is denoted Secondary Path. These marked paths have a double differentiator behaviour, since as input one has the voice coil displacement and as output the air acoustic pressure.

Both speakers are connected to a target computer with Simulink Real-time ${ }^{\circledR}$ environment through a pair of high definition power amplifiers and a data acquisition board. A second computer is used for development, design and operation with Matlab ${ }^{\circledR}$. The sampling frequency has been 


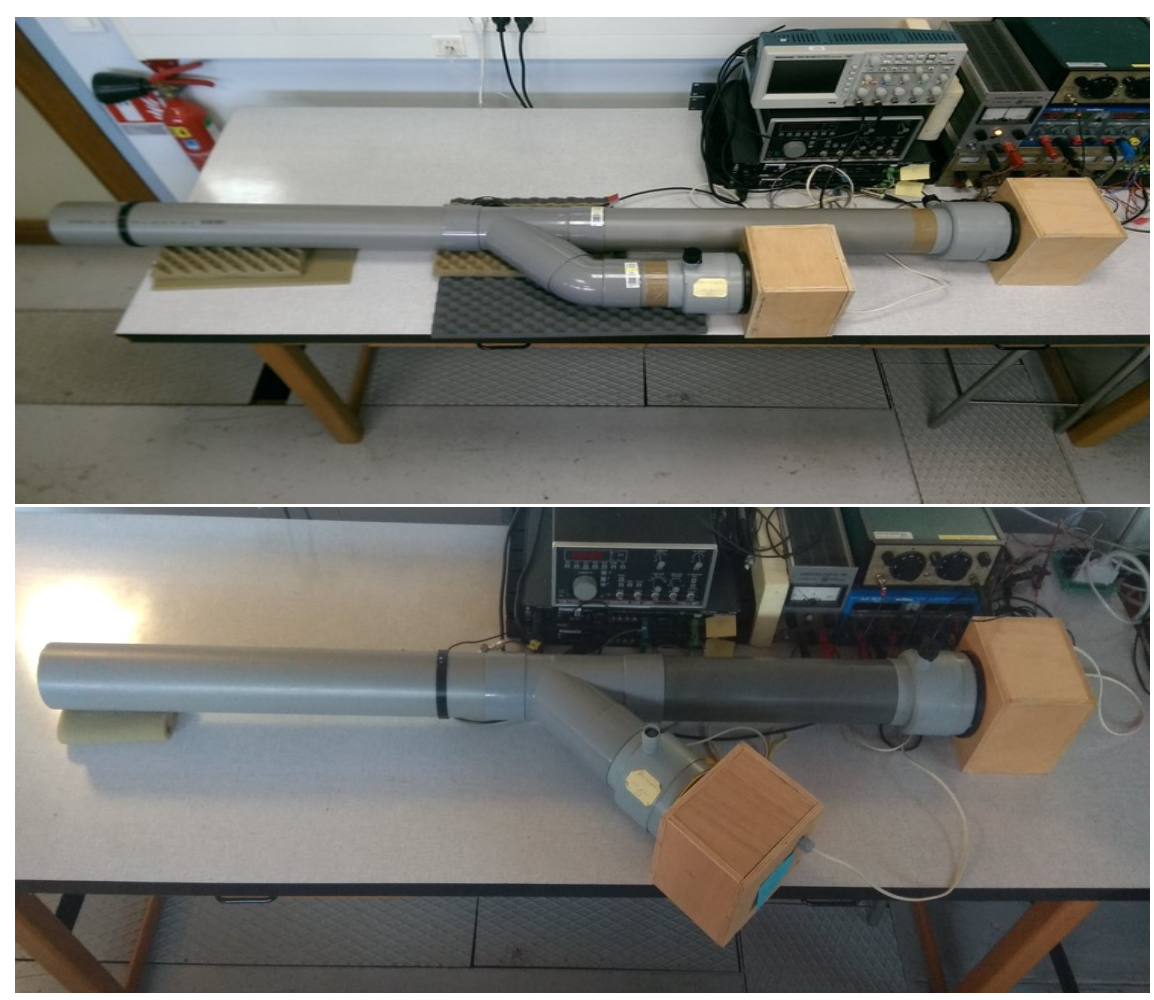

Figure 3: Duct active noise control test bench - Configuration Go (top), configuration G (bottom)

chosen in accordance with the recommendations given in [20]. Taking into account that disturbances up to $400 \mathrm{~Hz}$ need to be attenuated, a sampling frequency $f_{s}=2500 \mathrm{~Hz}$ has been chosen $\left(T_{s}=0.0004 \mathrm{sec}\right)$, i.e., approximately six times the maximum frequency to attenuate.

In this configuration, speakers are isolated inside wood boxes filled with special foam in order to create anechoic chambers and reduce the radiation effect. These boxes have dimensions $0.15 \mathrm{~m} \times 0.15 \mathrm{~m} \times 0.12 \mathrm{~m}$, giving a chamber volume of $2.7 \mathrm{~L}$.

\section{System structure}

The model of the real plant ${ }^{2}$ is denoted $G\left(q^{-1}\right)$ and is decribed by the transfer operator ${ }^{3}$,

$$
G\left(q^{-1}\right)=\frac{q^{-d} B^{\prime}\left(q^{-1}\right)}{A\left(q^{-1}\right)}=\frac{B\left(q^{-1}\right)}{A\left(q^{-1}\right)}
$$

\footnotetext{
${ }^{2}$ In active vibration and noise control, the plant is called "secondary path" or "compensation path"

${ }^{3}$ The complex variable $z^{-1}$ will be used for characterizing the system's behavior in the frequency domain and the delay operator $q^{-1}$ will be used for describing system's behavior in the time domain.
} 

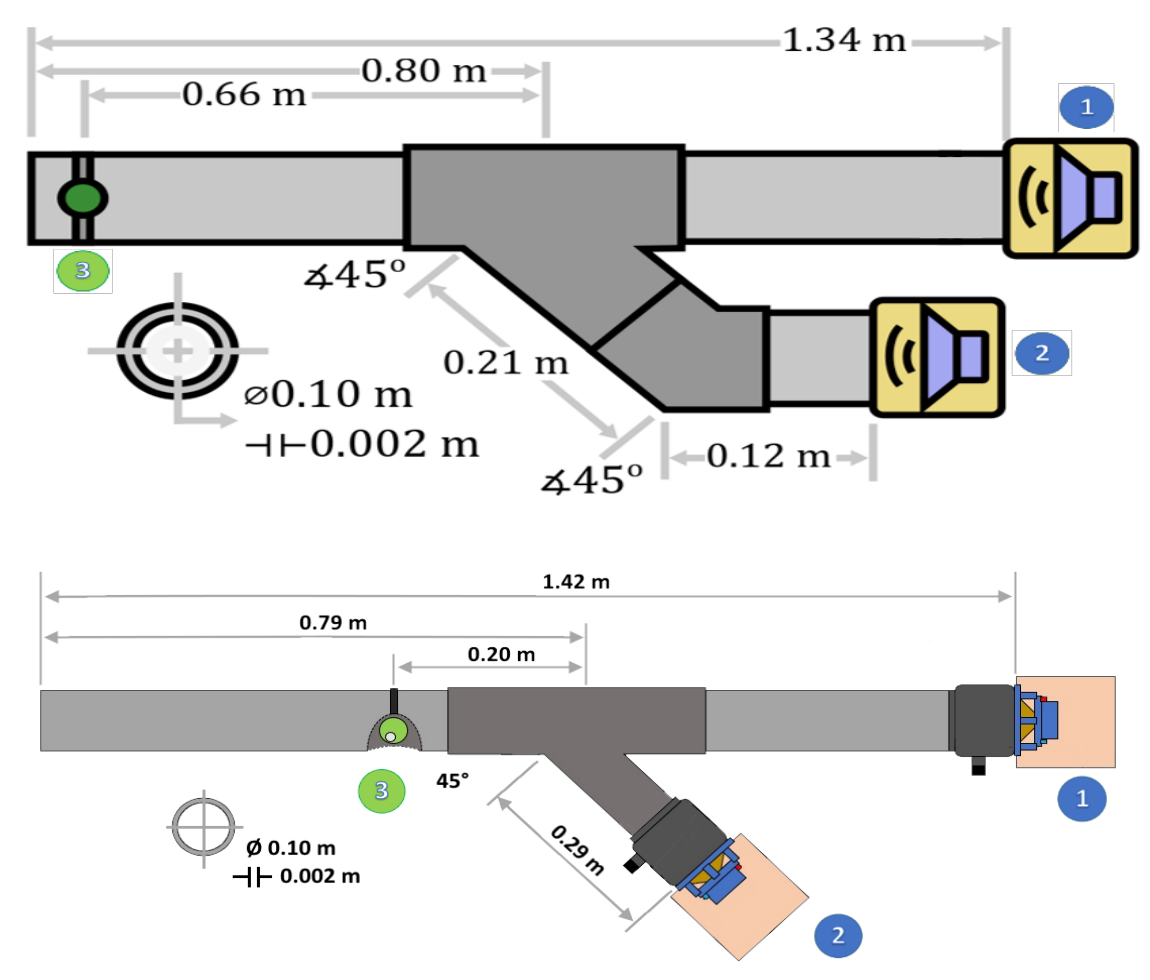

Figure 4: Duct active noise control test-bench dimensions - Go (top), G (bottom)

with:

$$
\begin{array}{r}
d=\text { plant pure time delay in number of sampling periods } \\
\qquad \begin{array}{r}
A=1+a_{1} q^{-1}+\cdots+a_{n_{A}} q^{-n_{A}} ; \\
B^{\prime}=b_{1} q^{-1}+\cdots+b_{n_{B}} q^{-n_{B}}=q^{-1} B^{*} ; \\
B^{* *}=b_{1}+\cdots+b_{n_{B}} q^{-n_{B}+1} ; \\
B=q^{-d} B^{\prime}\left(q^{-1}\right) ; \\
B^{*}=q^{-d} B^{*}\left(q^{-1}\right) ;
\end{array}
\end{array}
$$

The model of the compensation path used for controller design (design model) is denoted $G_{o}\left(q^{-1}\right)$ and is described by:

$$
G_{o}\left(q^{-1}\right)=\frac{q^{-d_{o}} B_{o}^{\prime}\left(q^{-1}\right)}{A_{o}\left(q^{-1}\right)}=\frac{B_{o}\left(q^{-1}\right)}{A_{o}\left(q^{-1}\right)}
$$

The secondary path models of the two configurations have been identified from experimental data using the methodology described in [6]. Fig. 5] shows the frequency characteristics of the identified models (magnitude and phase), and the phase lag of the two systems up to $250 \mathrm{~Hz}$ is given in Table 1. There are important differences between the two models. These characteristics present multiple resonances (low damped complex poles) $)^{4}$ and anti-resonances (low damped complex ze-

\footnotetext{
${ }^{4}$ The lowest damping is around 0.01 .
} 


\begin{tabular}{|c|c|c|c|c|c|c|c|c|c|c|}
\hline Freq. (Hz) & 70 & 90 & 110 & 130 & 150 & 170 & 190 & 210 & 230 & 250 \\
\hline System & -208 & -248 & -277 & -307 & -320 & -365 & -424 & -471 & -552 & -616 \\
\hline Phase $G_{o}\left(^{\circ}\right)$ & -178 & -229 & -262 & -294 & -329 & -357 & -382 & -477 & -530 & -551 \\
\hline Phase $G\left(^{\circ}\right)$ & 30 & 19 & 15 & 13 & -9 & 8 & 42 & -6 & 22 & 65 \\
\hline Phase $\left(G_{o}-G\right)\left(^{\circ}\right)$ & 30 &
\end{tabular}

Table 1: Phase $\left(^{\circ}\right)$ of the two systems

ros). The orders of the two models are summarized in Table 2. We will use the model of the secondary path of configuration Go (design model) as the model for the design of a Youla-Kucera adaptive feedback noise attenuation scheme to be applied to configuration $G$ (current plant). The experiments will be carried on configuration $\mathrm{G}$.

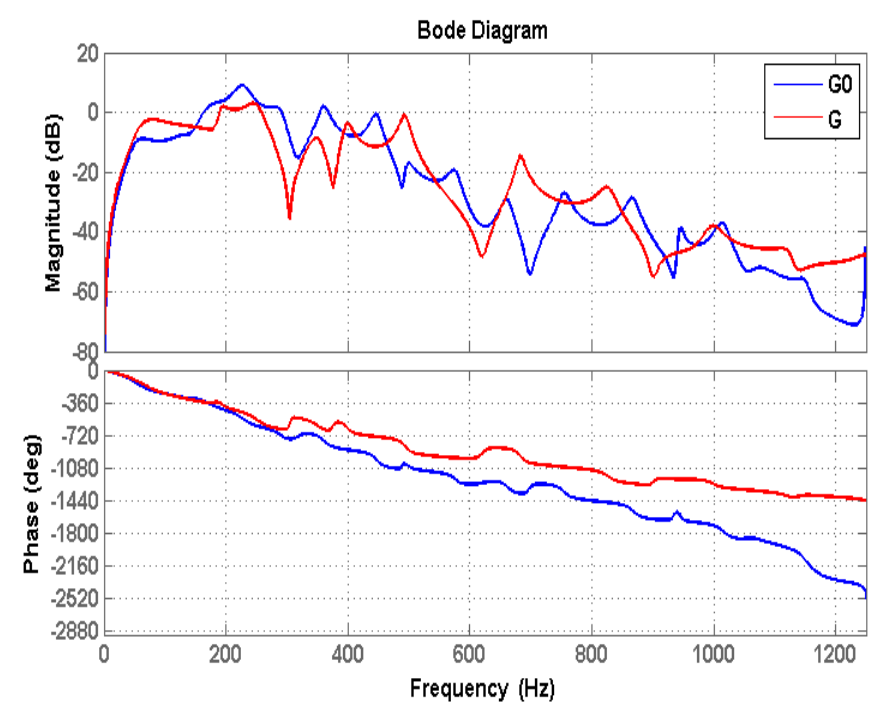

Figure 5: Bode diagrams of the nominal and uncertain plants

\begin{tabular}{|c|c|c|c|}
\hline Model & $n_{A}$ & $n_{B}$ & $d$ \\
\hline \hline Secondary path Go & 38 & 32 & 8 \\
\hline Secondary path G & 27 & 20 & 7 \\
\hline
\end{tabular}

Table 2: Orders of the identified models. 


\section{Controller Design}

\subsection{Closed-loop structure}

Let $\{u(t)\},\{y(t)\},\{d(t)\}$ be respectively the control input, the system output and the output disturbance sequences. The narrow band disturbance can be modeled as

$$
d(t)=\frac{N_{d}\left(q^{-1}\right)}{D_{d}\left(q^{-1}\right)} \delta(t)
$$

where $\delta$ is the Dirac impulse and $D_{d}\left(q^{-1}\right)$ is a polynomial with all his roots on the unit circle. If the real plant model is equal to the design model, one has

$$
y(t)=G_{0}\left(q^{-1}\right) u(t)+d(t)
$$

As shown in Fig. 1, the control structure is made of a disturbance observer providing an image $w(t)$ of the disturbance and a feedback structure yielding the signal $u(t)$. The disturbance observer output is given by:

$$
w(t)=-B_{0}\left(q^{-1}\right) u(t)+A_{0}\left(q^{-1}\right) y(t)=A_{0}\left(q^{-1}\right) d(t)
$$

For the purpose of this paper the Q-filter of Fig. 1 is considered to be a polynomial of the form

$$
Q\left(q^{-1}\right)=q_{0}^{Q}+q_{1}^{Q} q^{-1}+\cdots+q_{n_{Q}}^{Q} q^{-n_{Q}}
$$

In the general case, according to Fig. 1 a stabilizing R-S controller $C_{0}$ is employed such that

$$
C_{0}\left(q^{-1}\right)=\frac{R_{0}\left(q^{-1}\right)}{S_{0}\left(q^{-1}\right)}=\frac{R_{0}^{\prime}\left(q^{-1}\right) H_{R}\left(q^{-1}\right)}{S_{0}^{\prime}\left(q^{-1}\right) H_{S}\left(q^{-1}\right)}
$$

where $H_{R}\left(q^{-1}\right)$ and $H_{S}\left(q^{-1}\right)$ are some fixed parts imposed during the controller synthesis.

The polynomial defining the poles of the feedback system in the absence of the $\mathrm{Q}$ filter is given by:

$$
P_{0}\left(q^{-1}\right)=A_{0}\left(q^{-1}\right) S_{0}\left(q^{-1}\right)+R_{0}\left(q^{-1}\right) B_{0}\left(q^{-1}\right)
$$

The Q-filter combined with the nominal controller leads to the controller $C\left(q^{-1}\right)=\frac{R\left(q^{-1}\right)}{S\left(q^{-1}\right)}$ with

$$
u(t)=-\frac{R\left(q^{-1}\right)}{S\left(q^{-1}\right)} y(t)
$$

where

$$
\begin{array}{r}
R=R_{o}+A_{o} H_{R} H_{S} Q \\
S=S_{o}-B_{o} H_{R} H_{S} Q
\end{array}
$$

By simple computation, it can be shown that the poles of the closed loop remain unchanged in the presence of a $\mathrm{Q}$ filter having the structure given in Eq. (7). 
According to the internal model principle of Wohnam [1, 2], in order to reject asymptotically the disturbance $d(t)$, the controller $C$ must include in the polynomial $\mathrm{S}$ the denominator $D_{p}$ of the disturbance model and owing to Eqs. (4) and (11) one must have

$$
S=S^{\prime} D_{d}=S_{o}-H_{R} H_{S} B_{o} Q
$$

and the optimal value of $\mathrm{Q}$ is obtained by solving the Bezout equation:

$$
S^{\prime} D_{d}+H_{R} H_{S} B_{o} Q=S_{o}
$$

for $D_{d}\left(q^{-1}\right)=1+d_{1} q^{-1}+\cdots+d_{n_{D}} q^{-n_{D}}$. This Bezout equation 13 has a solution if $D_{d}$ and $H_{R} H_{S} q^{-d_{o}} B_{o}$ are coprime and a minimal solution is obtained for $n_{Q}=n_{D}-1$.

\subsubsection{The real plant differs with respect to the design model}

In the general case, the real plant $G\left(q^{-1}\right)=\frac{B\left(q^{-1}\right)}{A\left(q^{-1}\right)}$ differs from $G_{0}$. Consequently, the scheme of Fig. 1 is modified: the true secondary path is now $G$ instead of $G_{0}$, but the disturbance observer remains the one given by Eq. (6), and one has now

$$
y(t)=G\left(q^{-1}\right) u(t)+d(t)=\frac{B\left(q^{-1}\right)}{A\left(q^{-1}\right)} u(t)+d(t)
$$

In a first step, we will analyze this configuration in order to see if assuming that the model of the disturbance is known one can find a polynomial Q which leads to a stable closed loop system and allows asymptotic rejection of the disturbance.

Different structures for the central controller can be considered. Since both $G$ and $G_{o}$ are asymptotically stable and in order to avoid the design of controller stabilizing both $G$ and $G_{o}$, one considers in the sequel a simplification to the controller structure by imposing $R_{0}=0$ and $H_{S}=1$. Therefore, from Eqs. (10) and (11), the control signal becomes

$$
u(t)=-\frac{R}{S}=-\frac{A_{0} H_{R} Q}{S_{0}-B_{0} H_{R} Q} y(t)
$$

To express the control $\mathrm{u}(\mathrm{t})$ as a function of $\mathrm{w}(\mathrm{t})$, one obtains from Eq. (15):

$$
S_{0} u(t)=-H r Q\left[A_{0} y(t)-B_{0} u(t)\right]
$$

from which it results using Eq. (6) :

$$
u(t)=-\frac{H_{R}\left(q^{-1}\right)}{S_{0}\left(q^{-1}\right)} Q\left(q^{-1}\right) w(t)
$$

This control structure corresponds to Fig. 6 .

According to Eq. (14), the closed-loop polynomial including $G$ and the simplified version of the controller is

$$
P=A S_{0}+Q H_{R}\left(B A_{0}-B_{0} A\right)
$$




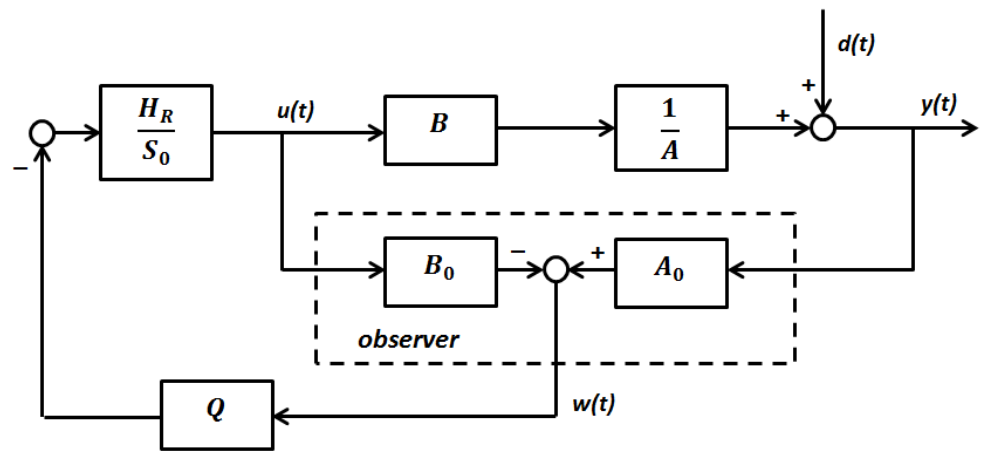

Figure 6: Linear controller structure for the case of known disturbances

which must have all its roots strictly inside the unit circle. In this case $\left(G \neq G_{0}\right)$, the signal $w(t)$ is expressed from $d(t)$ as:

$$
w(t)=\frac{A S_{0} A_{0}}{A S_{0}+Q H_{R}\left(B A_{0}-B_{0} A\right)} d(t)
$$

Derivation of this equation is given in Appendix $\mathrm{A}$.

The output sensitivity function from $d(t)$ to $y(t)$ is given in this case by

$$
y(t)=\frac{A S}{P} d(t)=\frac{A\left(S_{0}-B_{0} H_{R} Q\right)}{A S_{0}+Q H_{R}\left(B A_{0}-A B_{0}\right)} d(t)
$$

The derivation of this expression is given in Appendix $\mathrm{B}$. In the case of an uncertain plant a stability condition appears: The polynomial $A S_{0}+Q H_{R}\left(B A_{0}-B_{0} A\right)$ must have all its roots strictly inside the unit circle, and on the other hand the internal model condition (13) must be satisfied at the same time, in order to reject asymptotically $d(t)$. In case of an uncertain plant, two conditions appear: The polynomial $A S_{0}+Q H_{R}\left(B A_{0}-B_{0} A\right)$ must have all its roots strictly inside the unit circle for stability and the internal model condition (13) must be satisfied at the same time, in order to reject asymptotically $d(t)$. For a given order of the Q-filter, these two conditions may not be simultaneously met. In such a situation one can augment the order of $Q\left(q^{-1}\right)$. Non-minimal solution to Eq. (13) can be parametrized as $Q\left(q^{-1}\right)=\bar{Q}\left(q^{-1}\right)+V\left(q^{-1}\right) D_{D}\left(q^{-1}\right)$, [19] and Eq. (13) becomes

$$
\left(S^{\prime}-V B_{o} H_{R} H_{S}\right) D_{d}+H_{R} H_{S} B_{o}\left(\bar{Q}+V D_{d}\right)=S_{o}
$$

where $V\left(q^{-1}\right)$ is any polynomial (there is no need for it to be monic), and $\bar{Q}$ is the minimal solution of Eq. (13). The basic question is: does it exist a polynomial $Q\left(q^{-1}\right)$ with a degree $n_{Q}<\infty$ satisfying Eq. (21) and guaranteeing the closed-loop stability.

Under the hypothesis that $G_{0}$ and $G$ are stable and that $S_{0}$ has all its zeros strictly inside the unit circle, the sufficient conditions for the existence of a finite dimensional Q-filter which stabilizes the closed-loop and ensures the asymptotic rejection of the disturbance $d(t)$ are: 
1) At the disturbance frequencies $\omega_{j}$ corresponding to $D_{d}\left(e^{i \omega_{j}}\right)=0$, the transfer function

$$
\frac{A\left(z^{-1}\right)}{A_{0}\left(z^{-1}\right)} \frac{B_{0}\left(z^{-1}\right)}{B\left(z^{-1}\right)}-\frac{1}{2}
$$

is strictly positive real $5^{5}$

2) For all other frequencies the following inequality is verified

$$
\left|Q\left(e^{-i \omega}\right)\right|<\left|\frac{A\left(e^{-i \omega}\right)}{B\left(e^{-i \omega}\right) A_{0}\left(e^{-i \omega}\right)-A\left(e^{-i \omega}\right) B_{0}\left(e^{-i \omega}\right)}\right|\left|\frac{S_{0}\left(e^{-i \omega}\right)}{H_{R}\left(e^{-i \omega}\right)}\right|
$$

Derivation of conditions (22) and (23) is given in Appendix C. From this analysis, one concludes that we have to satisfy two conditions. The first condition (22) depends only upon the discrepancy of the two models and defines the frequency zones where asymptotic rejection of the disturbances can be achieved. This result can also be interpreted as the condition that $\frac{B_{o}\left(z^{-1}\right)}{A_{o}\left(z^{-1}\right)} \frac{A\left(z^{-1}\right)}{B\left(z^{-1}\right)}-\frac{1}{2}$ has a phase within $]-90^{\circ} ;+90^{\circ}$ [ in the frequency zone where asymptotic disturbance attenuation can be done.

The second condition indicates that one has to design $S_{0}$ and $H_{r}$ such that modulus of the right hand side of Eq. (23) is maximized in the frequencies regions outside the attenuation zone.

\subsection{Adaptive disturbance rejection}

In the presence of unknown narrow band disturbances the polynomial $D_{d}\left(q^{-1}\right)$ is unknown. In this situation one can consider a Q-filter with adjustable parameters:

$$
\hat{Q}\left(q^{-1}, t\right)=\hat{q}_{0}^{Q}(t)+\cdots \hat{q}_{n Q}^{Q}(t) q^{-n Q}
$$

and the objective is to find a parameter adaptation algorithm driving this parameters towards the values assuring asymptotic rejection of the disturbance and the stability of the system. We will follow up to certain extent the development procedure described in [2] but including from the beginning the presence of model uncertainties and the use of a $\mathrm{Q}$ filter of higher order than the minimal one used for the nominal case (when $G=G_{0}$ ). From Eq. (19) one can express d(t) as a function of $\mathrm{w}(\mathrm{t})$ and plugging this expression in Eq. (20) one gets:

$$
y(t)=\left(\frac{1}{A_{0}}-\hat{Q} \frac{H_{R} B_{0}}{A_{0} S_{0}}\right) w(t)
$$

where now the Q filter has been replaced by its estimation. Since the purpose of the control structure is to drive $y(t)$ towards 0 , it is the reasonable to define an a-posteriori prediction error $v(t+1)$ at time $t+1$, such that $v(t+1)=y(t+1)$ i.e.

$$
v(t+1)=\left(\frac{S_{0}}{A_{0} S_{0}}-\hat{Q} \frac{H_{R} B_{0}}{A_{0} S_{0}}\right) w(t+1)
$$

\footnotetext{
${ }^{5}$ This means that the poles of the transfer function are inside the unit circle and for all frequencies $\omega_{j}$ (i.e. the domain of operation) the real part is strictly positive real or equivalently, the phase lag is within $]-90^{\circ} ;+90^{\circ}$ [
} 
According to the internal model principle, the expression of $Q$ that satisfies the internal model principle is given by Eq. (13), and by using this expression in Eq. (26) one obtains

$$
v(t+1)=(Q-\hat{Q}) \frac{H_{R} B_{0}}{A_{0} S_{0}} w(t+1)+p(t+1)
$$

where

$$
p(t+1)=\frac{S^{\prime} D_{d}}{A S_{0}+Q H r\left(B A_{0}-B_{0} A\right)} \cdot \frac{N_{d}}{D_{d}} \delta(t)
$$

is a vanishing term assuming that the optimal Q stabilizes also the system. See also [2].

One can define:

$$
\begin{array}{r}
w_{1}(t)=\frac{1}{A_{0}} w(t) \\
w_{2}(t)=\frac{B_{0}\left(q^{-1}\right) H_{R}\left(q^{-1}\right)}{A_{0}\left(q^{-1}\right) S_{0}\left(q^{-1}\right)} w(t+1) \\
\hat{\mathbf{w}}^{\mathbf{T}}(\mathbf{t})=\left[\hat{\mathbf{q}}_{\mathbf{0}}^{\mathbf{Q}}(\mathbf{t}), \hat{\mathbf{q}}_{\mathbf{1}}^{\mathbf{Q}}(\mathbf{t}), \cdots \hat{\mathbf{q}}_{\mathbf{n}_{\mathbf{Q}}}^{\mathbf{Q}}(\mathbf{t})\right] \\
\mathbf{w}^{T}(t)=\left[\mathbf{q}_{\mathbf{0}}^{\mathbf{Q}}(\mathbf{t}), \mathbf{q}_{\mathbf{1}}^{\mathbf{Q}}(\mathbf{t}), \cdots \mathbf{q}_{\mathbf{n}_{\mathbf{Q}}}^{\mathbf{Q}}(\mathbf{t})\right] \\
\mathbf{r}^{T}(t)=\left[w_{2}(t), w_{2}(t-1), \cdots w_{2}\left(t-n_{Q}\right)\right]
\end{array}
$$

The a-priori adaptation error is given by:

$$
v^{\circ}(t+1)=w_{1}(t+1)-\hat{\mathbf{w}}^{T}(t) \mathbf{r}(t)
$$

and the a-posteriori adaptation error is given by:

$$
v(t+1)=\frac{v^{\circ}(t+1)}{1+\mathbf{r}^{T}(t) \mathbf{F}(t) \mathbf{r}(t)}
$$

The a-posteriori adaptation error (27) can be expressed now under the form

$$
\varepsilon(t+1)=H\left(q^{-1}\right)[\mathbf{w}-\hat{\mathbf{w}}(t+1)]^{T} \mathbf{r}(t)
$$

where $H\left(q^{-1}\right)=1$.

In order to estimate the coefficients of $\hat{Q}\left(q^{-1}, t\right)$, it is natural to use a Parameter Adaptation Algorithm (PAA). Taking into account the fact that the order of the polynomial $\hat{Q}$ is higher than the minimal order required by the IMP, the parameter adaptation algorithm proposed in [9] has to be completed with a projection of the estimated parameter vector on a bounded domain in order to prove the stability of the adaptive control scheme (see [9] p.340). Based on stability considerations, a general form for the PAA has been proposed in [9]. It can be expressed using the formalism 
of [21] as:

$$
\begin{aligned}
& \hat{\mathbf{w}}(t+1)=\hat{\mathbf{w}}_{p}(t)+\mathbf{F}(t) \mathbf{r}(t) v(t+1) \\
& v(t+1)=\frac{v^{\circ}(t+1)}{1+\mathbf{r}^{T}(t) \mathbf{F}(t) \mathbf{r}(t)} \\
& \mathbf{F}(t+1)=\frac{1}{\lambda_{1}(t)}\left[\mathbf{F}(t)-\frac{\mathbf{F}(t) \mathbf{r}(t) \mathbf{r}^{T}(t) \mathbf{F}(t)}{\frac{\lambda_{1}(t)}{\lambda_{2}(t)}+\mathbf{r}^{T}(t) \mathbf{F}(t) \mathbf{r}(t)}\right] \\
& 1 \geq \lambda_{1}(t)>0 \quad ; \quad 0 \leq \lambda_{2}(t)<2 \quad ; \quad F(0)>0
\end{aligned}
$$

where

$$
\begin{array}{ll}
\hat{\mathbf{w}}^{\prime}(t)=F(t)^{-1 / 2} \hat{\mathbf{w}}(t) & \\
\hat{\mathbf{w}}_{p}(t)=\hat{\mathbf{w}}^{\prime}(t) & \text { if } \hat{\mathbf{w}}^{\prime}(t) \in \mathscr{D}^{\prime} \\
\hat{\mathbf{w}}_{p}^{\prime}(t)=\perp \text { proj of } \mathbf{w}^{\prime}(t) \text { on } \mathscr{D}^{\prime} & \text { if } \mathbf{w}^{\prime}(t) \notin \mathscr{D}^{\prime} \\
\hat{\mathbf{w}}_{p}(t)=F(t)^{1 / 2} \hat{\mathbf{w}}_{p}^{\prime}(t) &
\end{array}
$$

The projection domain $\mathscr{D}^{\prime}$ is defined as follows

$$
\hat{\mathbf{w}} \in \mathscr{D}, \quad \hat{\mathbf{w}}^{\prime}(t)=F(t)^{-1 / 2} \hat{\mathbf{w}}(t) \in \mathscr{D}^{\prime}
$$

where the projection domain $\mathscr{D}$ is such that:

$$
\mathscr{D}: \quad\|\hat{\mathbf{w}}(t)\|_{2}^{2}<\mathscr{R}<\infty
$$

The adaptive control structure is given in Fig. 7. The stability analysis of the full system is discussed in Appendix D.

In practice, in order to assure the alertness of the adaptation with respect to possible variations of the disturbance characteristics, two particular choices for the adaptation gain are used 6 .

- Constant trace: for a constant ratio $\lambda_{1}(t) / \lambda_{2}(t), \lambda_{1}(t)$ is chosen such that the trace of the adaptation gain matrix $F(t)$ remain constant $\left(\operatorname{trace} F(t)=\right.$ trace $\left.F_{0}\right)$

- Constant gain: $\lambda_{1}(t)=1, \lambda_{2}(t)=0$ and therefore $F(t)=F_{0}$ (usually $F_{0}=\alpha I, \alpha>0$ )

Remark: When using a constant adaptation gain, the change of coordinates introduced in Eqs. (37) and (38) is no more necessary (see [9], p.340-343) and Eqs. (37) and (38) are replaced by:

$$
\begin{gathered}
\hat{\mathbf{w}}_{p}(t)=\hat{\mathbf{w}}(t) \quad \text { if } \hat{\mathbf{w}}(t) \in \mathscr{D} \\
\hat{\mathbf{w}}(t)=\perp \text { proj of } \mathbf{w}(t) \text { on } \mathscr{D} \quad \text { if } \hat{\mathbf{w}}(t) \notin \mathscr{D}
\end{gathered}
$$

\footnotetext{
${ }^{6}$ For other options see [9]
} 


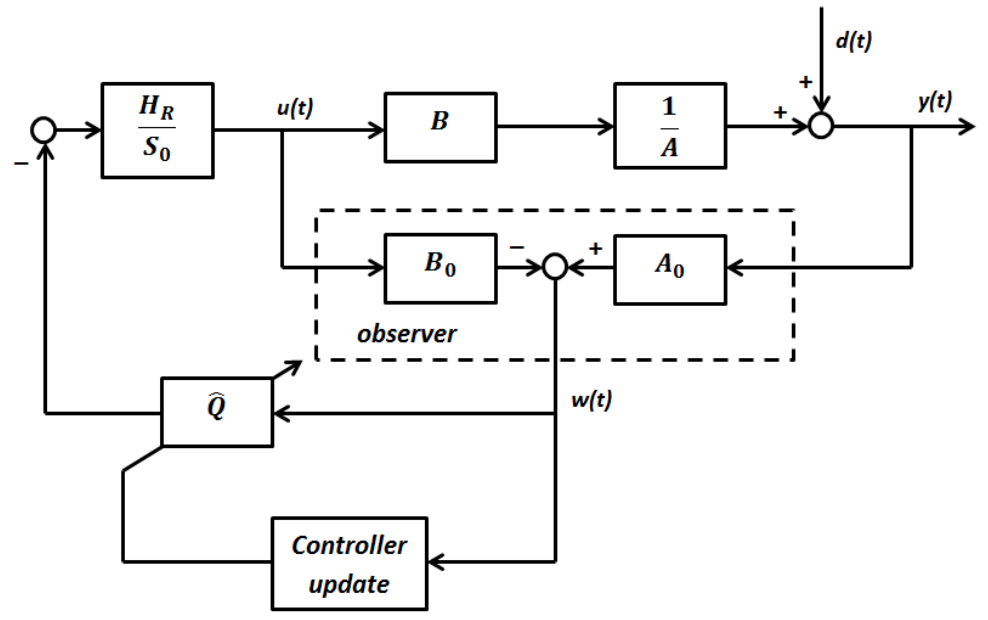

Figure 7: Adaptive control structure

This drastically simplifies implementation of the algorithm. This simplification is also used for the case of a constant trace adaptation gain.

Remark: The orthogonal projection, which gives the closest set of parameters to the estimated parameters at instant $\mathrm{t}$ and which are inside the domain $\mathscr{D}^{\prime}$, can be replaced by any set of parameters inside the domain $\mathscr{D}^{\prime}$ (sub optimal solution) and in particular by the initial value of the estimated parameters (usually set at 0 ). This simplifies computations and has little impact on the performance since the adaptation transients can be very fast. For more details see (see [9], p.341).

In practice a bound on the parameters of polynomial $Q$ is fixed and when one of the parameters hits the bound, the algorithm is restarted with all the parameters at 0 (i.e. one takes $\hat{\mathbf{w}}_{p}(t)=0$ in Eq. 37).

\section{Tuning of the filter $\mathrm{Hr} / \mathrm{So}$}

Firstly, it is necessary to check the frequency band for which a disturbance rejection is feasible, or in other words the band where condition (22) is satisfied. For this purpose one plots the phase of $\frac{B_{0}\left(e^{-i \omega}\right) A\left(e^{-i \omega}\right)}{B\left(e^{-i \omega}\right) A_{0}\left(e^{-i \omega}\right)}-\frac{1}{2}$ as a function of $\omega$, which should be within $]-90^{\circ} ;+90^{\circ}$. In the specific example of this paper, the corresponding graph is displayed in Fig. 8 and one concludes that an adaptive rejection of the disturbance is feasible roughly up to $250 \mathrm{~Hz}$.

In order to design the filter $\frac{H_{R}\left(q^{-1}\right)}{S_{0}\left(q^{-1}\right)}$, it is useful to plot $\left|\frac{A\left(e^{-i \omega}\right)}{B\left(e^{-i \omega}\right) A_{0}\left(e^{-i \omega}\right)-A\left(e^{-i \omega}\right) B_{0}\left(e^{-i \omega}\right)}\right|$, see Fig. 9 (top). In order to guarantee condition (23) for the largest possible family of $\mathrm{Q}$ filters, the product $\left|\frac{A\left(e^{-i \omega}\right)}{B\left(e^{-i \omega}\right) A_{0}\left(e^{-i \omega}\right)-A\left(e^{-i \omega}\right) B_{0}\left(e^{-i \omega}\right)}\right|\left|\frac{S_{0}\left(e^{-i \omega}\right)}{H_{R}\left(e^{-i \omega}\right)}\right|$ must be maximized, and for this purpose the magnitude of $\frac{S_{0}\left(q^{-1}\right)}{H_{R}\left(q^{-1}\right)}$ is increased as much as possible at the frequencies where the magnitude of $\frac{A\left(q^{-1}\right)}{\left.B\left(q^{-1}\right) A_{0}\left(q^{-1}\right)\right)-A\left(q^{-1}\right) B_{0}\left(q^{-1}\right)}$ is low. A solution consists in choosing the filter $\frac{H_{R}\left(q^{-1}\right)}{S_{0}\left(q^{-1}\right)}$ as a product of 

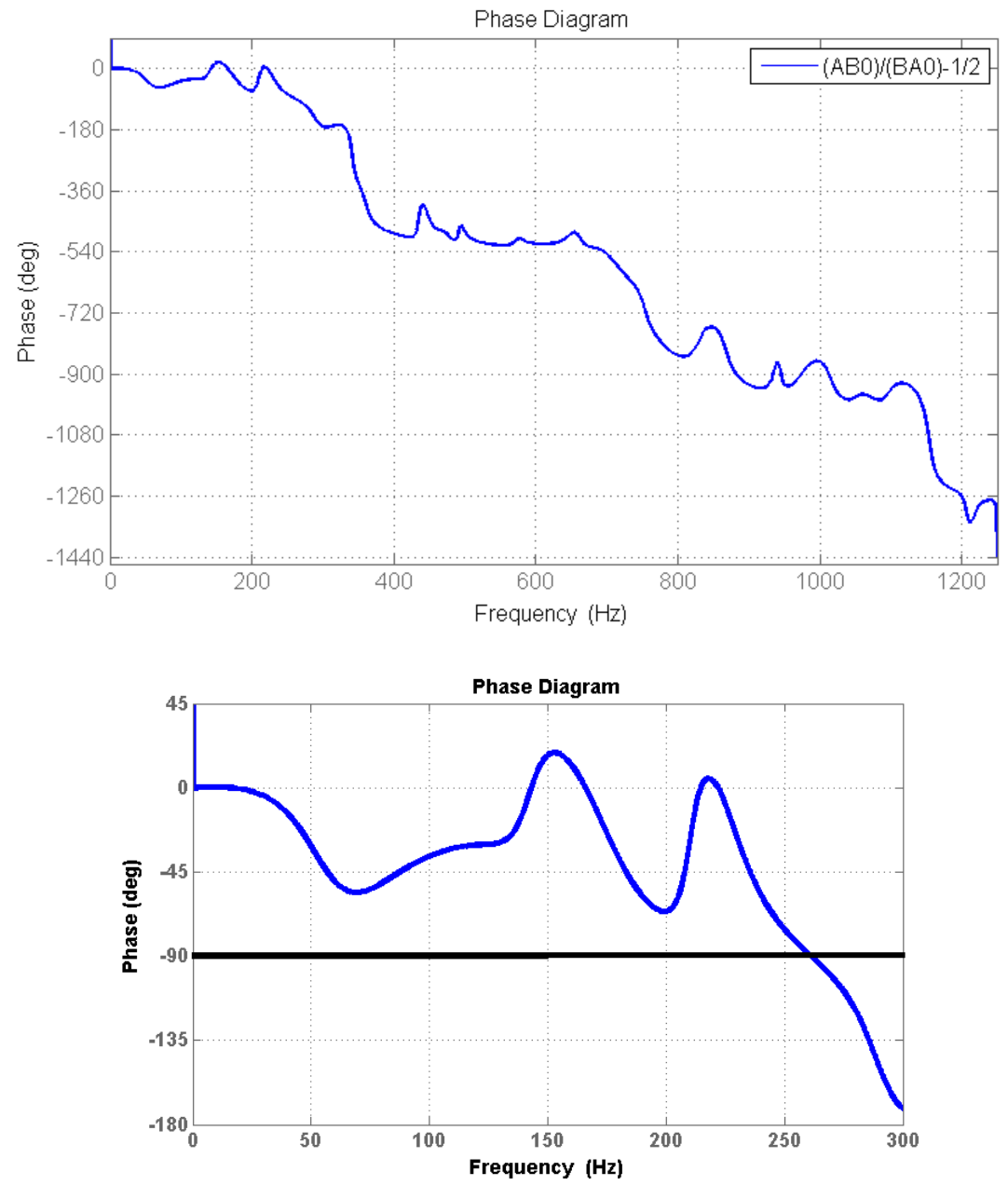

Figure 8: Phase of $\frac{B_{0}\left(z^{-1}\right) A\left(z^{-1}\right)}{A_{0}\left(z^{-1}\right) B\left(z^{-1}\right)}-\frac{1}{2}$ from 0 to $1250 \mathrm{~Hz}$ (top), from 0 to $300 \mathrm{~Hz}$ (bottom)

$n_{k}$ second order filters:

$$
\frac{H_{R}\left(q^{-1}\right)}{S_{0}\left(q^{-1}\right)}=\prod_{k=1}^{n_{k}} \frac{L_{N k}\left(q^{-1}\right)}{L_{D k}\left(q^{-1}\right)}
$$

where each filter $\frac{L_{N k}\left(q^{-1}\right)}{L_{D k}\left(q^{-1}\right)}$ results from discretization of a continuous resonant filter at the frequency $\omega_{k}$ for $k \leq n_{k}-1$ :

$$
\frac{\frac{s^{2}}{w_{k}^{2}}+2 \zeta_{N k} s+1}{\frac{s^{2}}{w_{k}^{2}}+2 \zeta_{D k} s+1}
$$

with $0 \leq \zeta_{N k}<1,0<\zeta_{D k} \leq 1$ and $\zeta_{N k}<\zeta_{D k}$. Moreover, $\frac{L_{N n_{k}}\left(q^{-1}\right)}{L_{D n_{k}}\left(q^{-1}\right)}$ has two zeros at +1 and -1 and two poles at 0 , in order to open the loop at $0 \mathrm{~Hz}$ (the system has no gain at this frequency and it is not reasonable to send a dc signal) and at the Nyquist frequency (for reducing the gain of the 
filter in high frequencies).

As a result, the magnitude of $\frac{S_{0}\left(q^{-1}\right)}{H_{R}\left(q^{-1}\right)}$ shows resonances at frequencies $\omega_{k}$. Fig. 9 (middle) displays the magnitude of this filter for this application. The first resonance frequency is equal to $225 \mathrm{~Hz}$, and the magnitude of $\frac{S_{0}\left(q^{-1}\right)}{H_{R}\left(q^{-1}\right)}$ for frequencies between $80 \mathrm{~Hz}$ and $210 \mathrm{~Hz}$ is left sufficiently low. For frequencies higher than $225 \mathrm{~Hz}$ a series of resonances guarantee that this magnitude is sufficiently high. Fig. 9 (bottom), presents the frequency characteristics of the right part of Eq. 23). One can see the effect of the filter $H_{R} / S_{o}$.
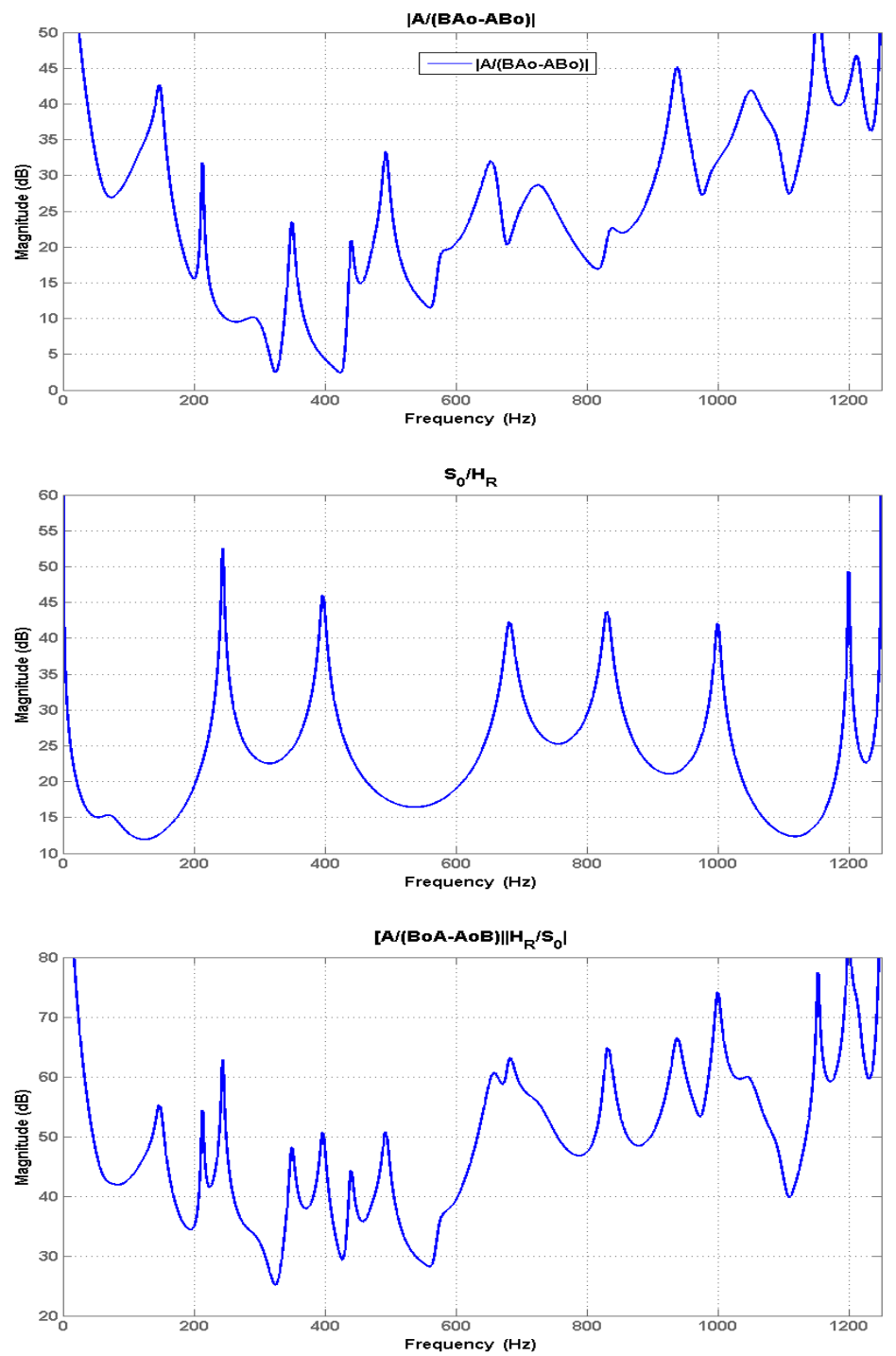

Figure 9: Magnitude of $\frac{A\left(q^{-1}\right)}{B\left(q^{-1}\right) A o\left(q^{-1}\right)-A\left(q^{-1}\right) B o\left(q^{-1}\right)}$ (top), Magnitude for $\frac{S_{0}\left(q^{-1}\right)}{H_{R}\left(q^{-1}\right)}$ (middle), Magintude of $\frac{A\left(q^{-1}\right)}{B\left(q^{-1}\right) A o\left(q^{-1}\right)-A\left(q^{-1}\right) B o\left(q^{-1}\right)} \frac{S_{0}\left(q^{-1}\right)}{H_{R}\left(q^{-1}\right)}$ (bottom) 


\section{Experimental Results}

The objective of the experimental validation is to assess the performance of the overparametrized YK adaptive feedback scheme on the configuration $G$ using the model of the configuration Go for implementing the YK observer (uncertainty case)

\subsection{Performance on configuration $G$ when using model Go}

The performance will be evaluated using: 1) a single sinusoidal disturbance, 2) two sinusoidal disturbances with very close frequencies (interference phenomenon), 3) two, three and four sinusoidal disturbances with distinct frequencies.

This evaluation has been done over the frequency range of operation. For all the experiments a constant adaptation gain algorithm $(F=\alpha I)$ is used with a value of the adaptation gain (step size) $\alpha=30 / 1.73$ except for the case of four sinusoidal disturbances where the constant trace algorithm is used with an equivalent adaptation gain by parameter (step size) of 30 .

\subsubsection{The case of a single sinusoidal disturbance}

For the case of a single sinusoid it was found that using a $\mathrm{Q}$ filter with $\mathrm{nQ}=15$ (16 parameters), good performance can be obtained over a range of operation between $80 \mathrm{~Hz}$ and $180 \mathrm{~Hz}$. Use of $n Q<11$ (12 parameters) leads to instabilities for almost all the frequencies within this range. The attenuation in steady state 7 for various frequencies of the disturbance are summarized in row 2 of Table 3 . Fig. 10 shows the time response for the rejection of a single $160 \mathrm{~Hz}$ sinusoidal

\begin{tabular}{ccccccccc}
\hline Frequency (Hz) & 70 & 80 & 120 & 160 & 180 & 190 & 210 & 220 \\
\hline Model Go, 16 par.(dB) & 45.64 & 69.04 & 61.89 & 81.05 & 88.41 & Oscil. & Oscil & Oscil. \\
\hline Model Go, 30 par.(dB) & 67.02 & 74.84 & 74.84 & 77.98 & 90.92 & 90.44 & 77.71 & 76.42. \\
\hline Model Go, 60 par.(dB) & 69.04 & 75.84 & 72.94 & 78.86 & 90.42 & 93.92 & 84.95 & 81.34. \\
\hline
\end{tabular}

Table 3: Steady state attenuation for a single sinusodal noise disturbance on configuration G

disturbance. The system operates in open loop for the first $5 \mathrm{~s}$. Fig. 11 shows the power spectral density (PSD) of the residual noise in open loop and in closed loop for the same disturbance (the PSD is evaluated over an horizon of 3s). Fig. 12 shows the response of the residual noise for a sequence of step changes in the frequency of the disturbance around $160 \mathrm{~Hz}$. The system operates in open loop for $5 \mathrm{~s}$ and then at $\mathrm{t}=50 \mathrm{~s}$ a step of $-10 \mathrm{~Hz}(150 \mathrm{~Hz})$ is applied. Then the system returns to the nominal frequency at $t=100 \mathrm{~s}$ and at $\mathrm{t}=150 \mathrm{~s}$ a step of $+10 \mathrm{~Hz}(170 \mathrm{~Hz})$ is applied. The corresponding evolution of the parameters is shown in Fig. 13. When using a Q filter with 16 parameters $(\mathrm{nQ}=15)$ oscillations occur for sinusoidal disturbances with frequencies equal or higher than $190 \mathrm{~Hz}$. However augmenting the size of the Q filter to 30 parameters $(\mathrm{nQ}=29)$ one can go up to $220 \mathrm{~Hz}$. The steady state attenuation for various frequencies of the disturbance for $n Q=29$ can be found in Table 3, row 3. Augmenting the size of the $\mathrm{Q}$ filter to $\mathrm{nQ}=59$

\footnotetext{
${ }^{7}$ Attenuation is defined as the ratio between the variance of the residual noise in open loop and the variance of the residual noise in closed loop
} 


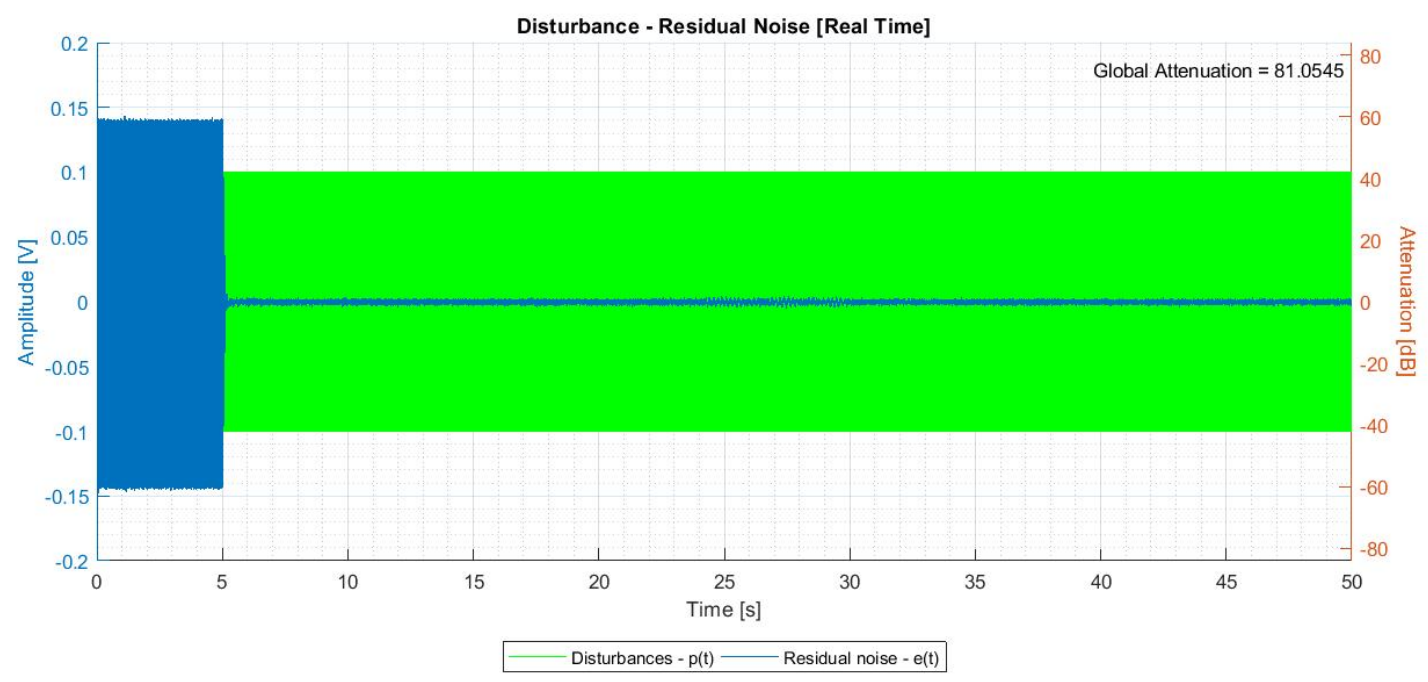

Figure 10: Evolution of the residual noise for a $160 \mathrm{~Hz}$ sinusodal disturbance (16 parameters).

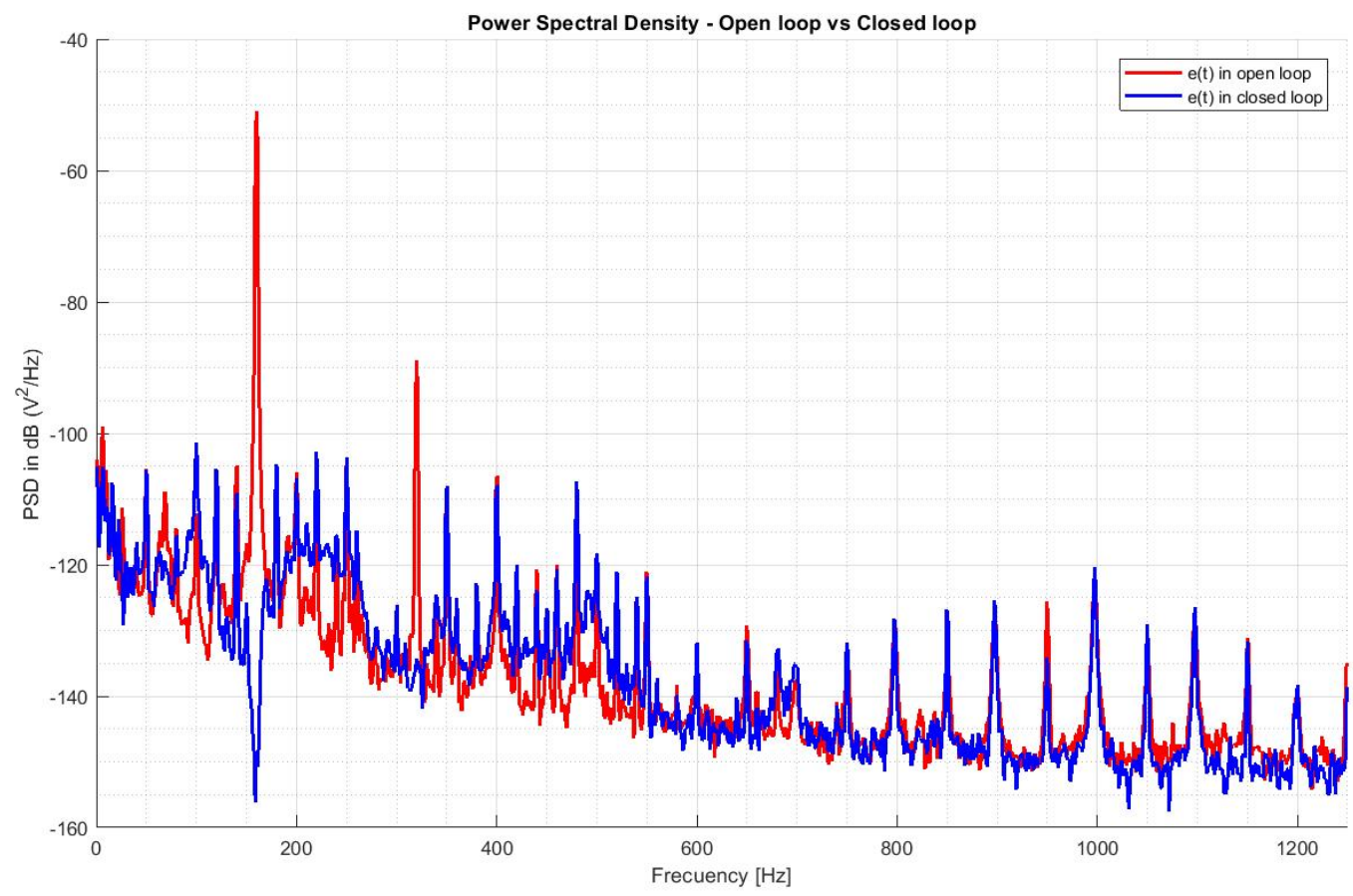

Figure 11: Power Spectral density of the residual noise in open loop and in closed loop for a $160 \mathrm{~Hz}$ sinusoidal disturbance (16 parameters) .

(60 parameters) allows to improve performance at the border of the operation region but does not allow to go up to higher frequencies. The steady state attenuation for this case is summarized in 


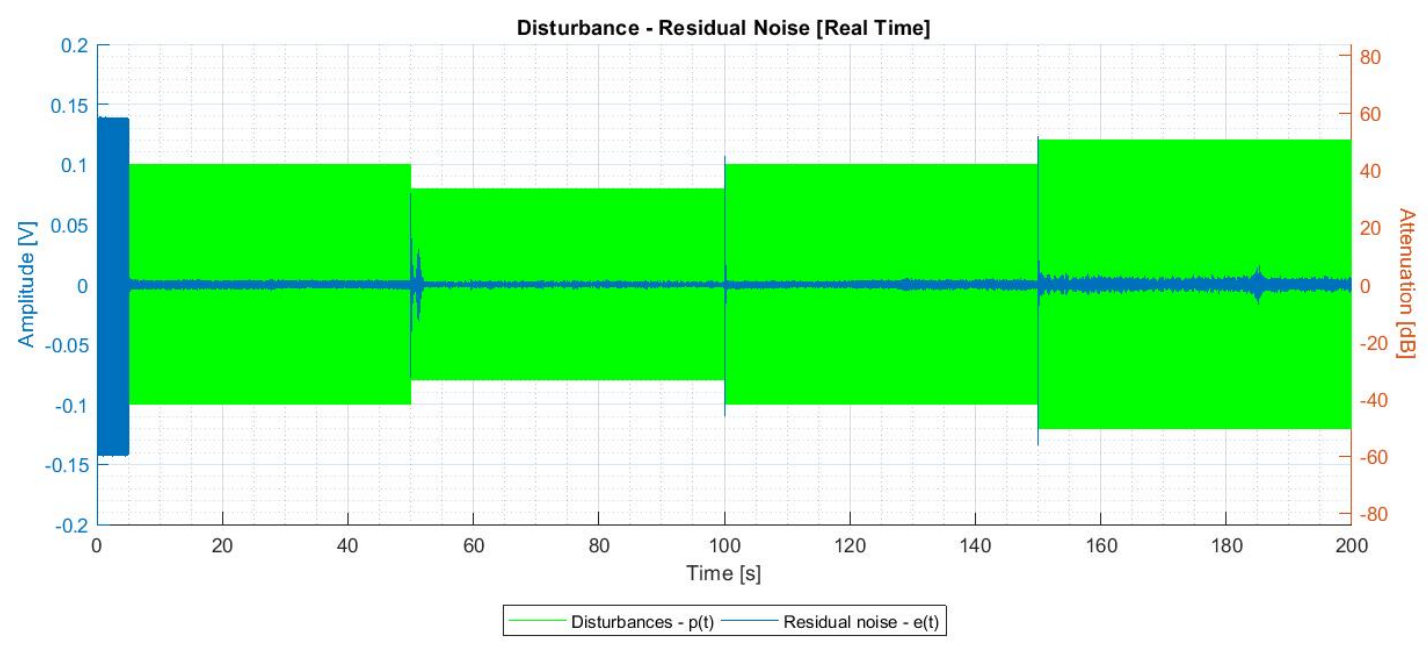

Figure 12: Time response of the residual noise for step changes in the frequency of the disturbance around $160 \mathrm{~Hz}$ (16 parameters)

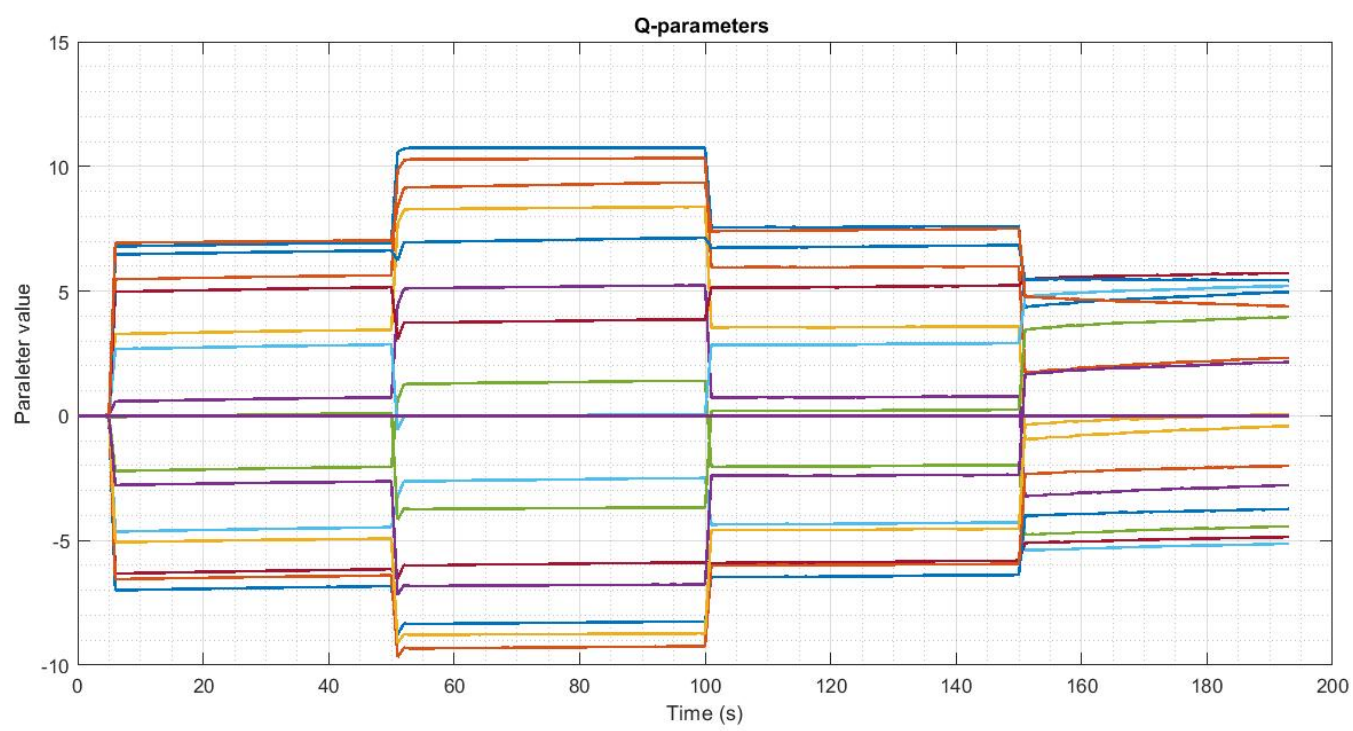

Figure 13: Evolution of the parameters for step changes in the frequency of the disturbance around $160 \mathrm{~Hz}(16$ parameters)

Table 3, row 4.

\subsubsection{Adaptive attenuation of interferences}

Fig. 14 shows the capability of the control scheme to strongly attenuate interference (interference occurs when two sinusoidal disturbances have very close frequencies). A couple of sinusoids at $140 \mathrm{~Hz}$ and $140.5 \mathrm{~Hz}$ is applied. Then at $\mathrm{t}=50 \mathrm{~s}$ one switches to $130 \mathrm{~Hz}$ and $130.2 \mathrm{~Hz}$, at $\mathrm{t}=100$ $\mathrm{s}$ one returns to $140 \mathrm{~Hz}$ and $140.5 \mathrm{~Hz}$ and at $\mathrm{t}=150 \mathrm{~s}$ one switches to $150 \mathrm{~Hz}$ and $150.3 \mathrm{~Hz}$. The 
system operates in open loop for the first $5 \mathrm{~s}$. The corresponding evolution of the parameters (16 parameters) is shown in Fig. 15

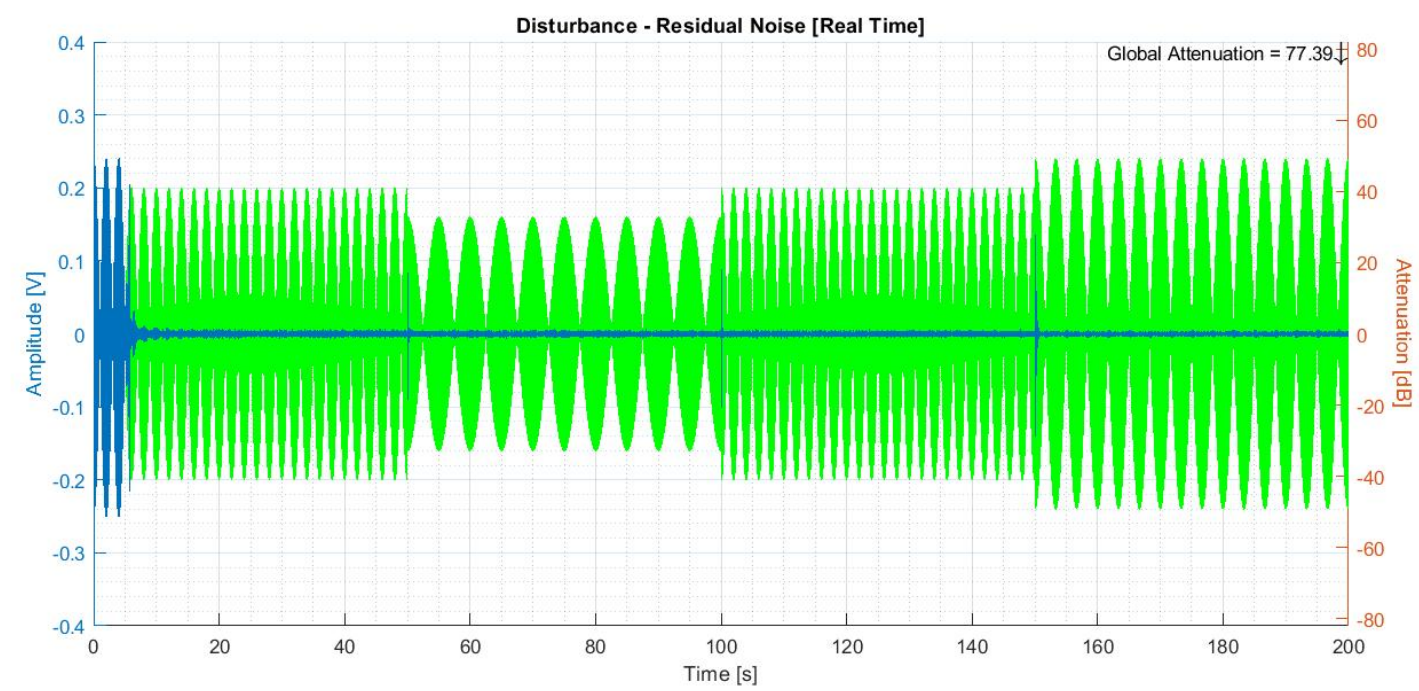

Figure 14: Time response of the residual noise for step changes in the frequencies of an interference phenomenon

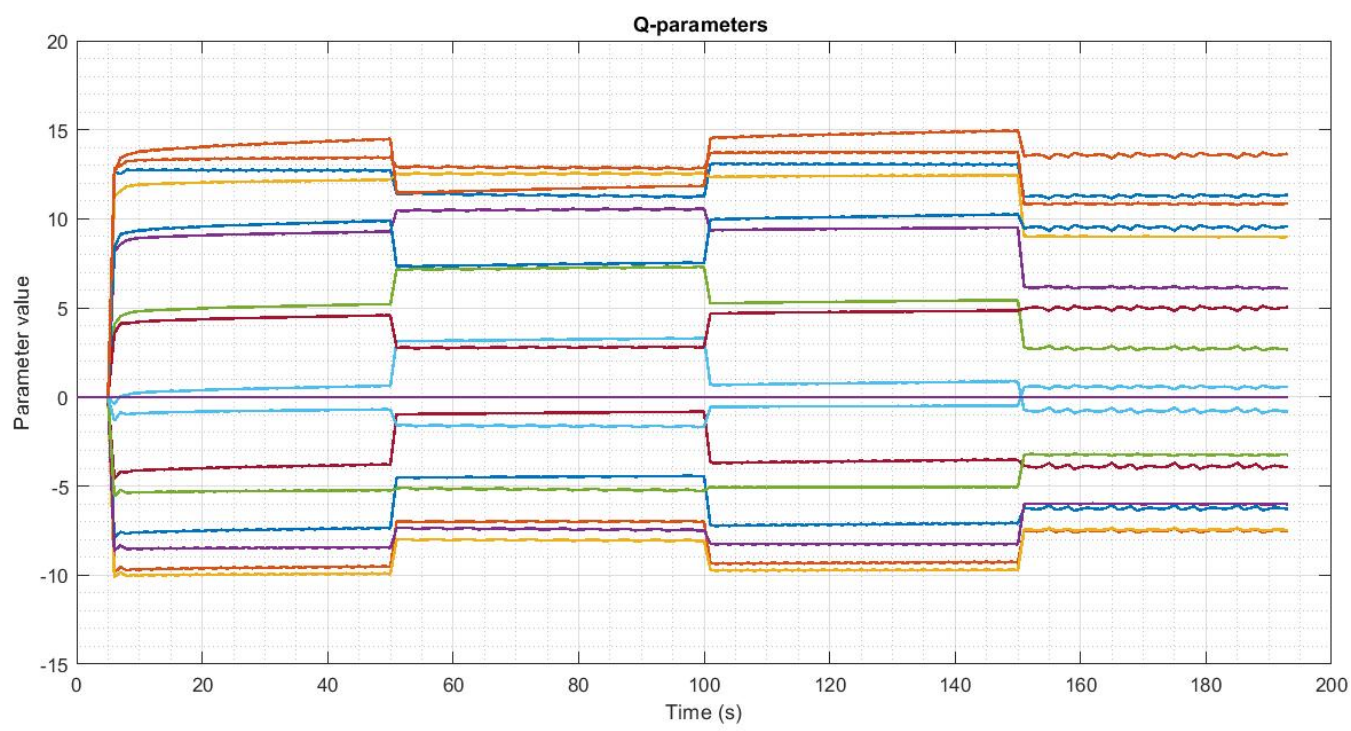

Figure 15: Evolution of the parameters for step changes in the frequencies of an interference phenomenon (16 parameters)

\subsubsection{The case of 2 sinusoidal disturbances of distinct frequencies}

To asymptotically reject the effect of two simultaneous sinusoidal disturbances with distinct frequencies and to assure simultaneously the stability of the system, the dimension of the Q filter 


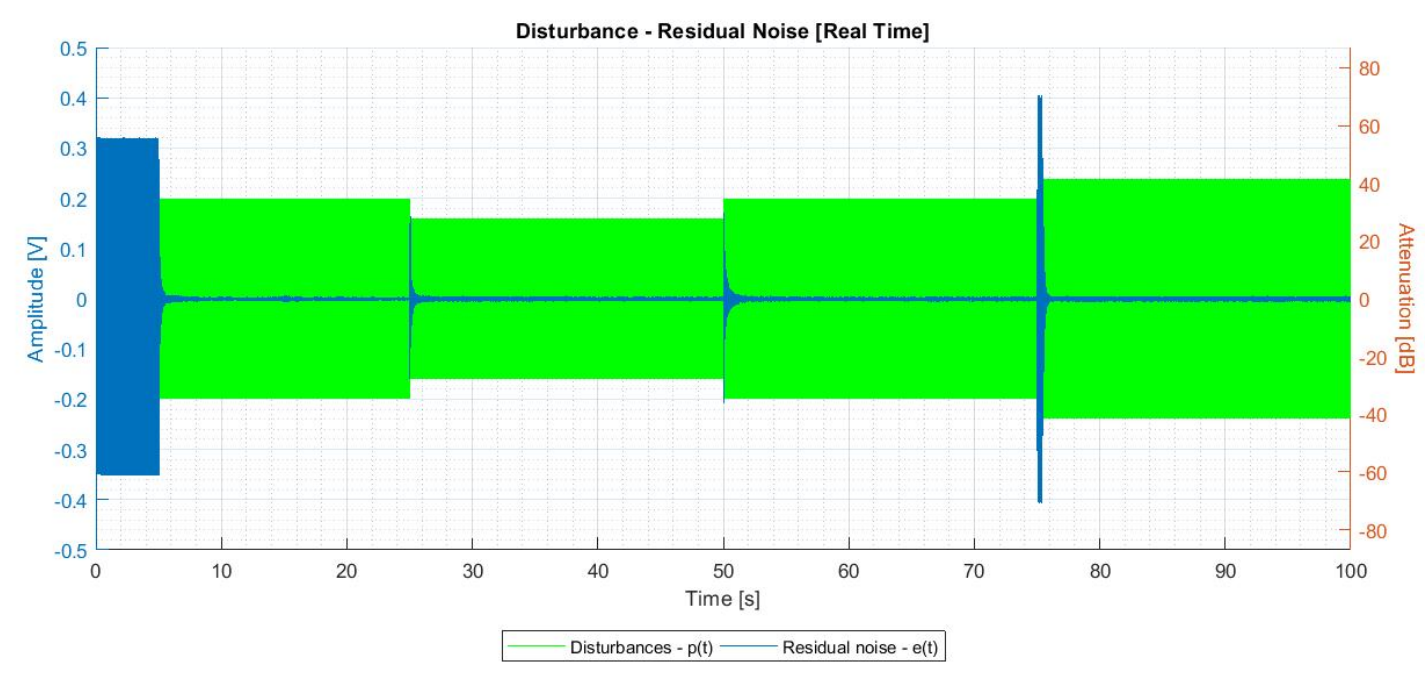

Figure 16: Time response of the residual noise for step changes in the frequency of two simultaneous sinusoidal disturbances around $80 \mathrm{~Hz}$ and $180 \mathrm{~Hz}$

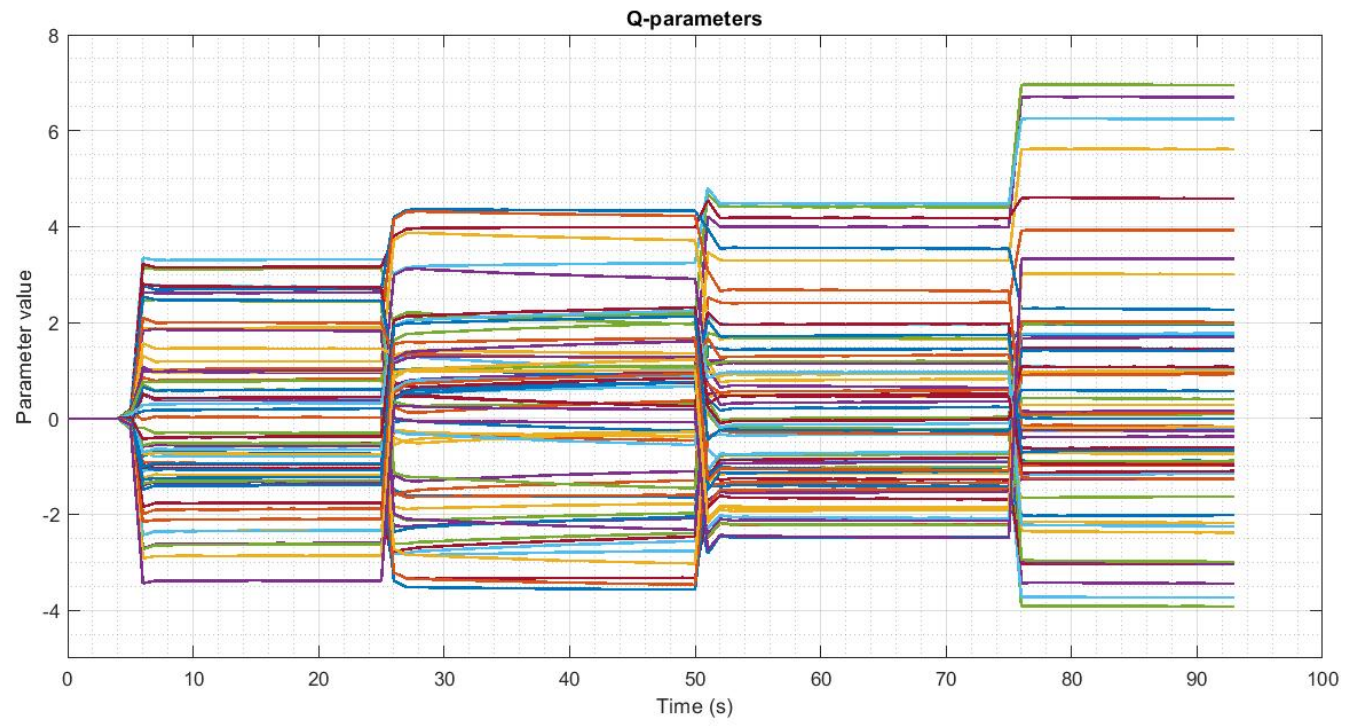

Figure 17: Evolution of the parameters for step changes in the frequency of two simultaneous sinusoidal disturbances around $80 \mathrm{~Hz}$ and $180 \mathrm{~Hz}$

should be significantly augmented. For $n Q=59$ (60 parameters) it is possible to assure a stable operation of the system for any combination of frequencies in the range $70 \mathrm{~Hz}$ to $190 \mathrm{~Hz}$. Fig. 16 gives the time response for a sequence of steps on the frequencies of two sinusoidal disturbances. The initial frequencies of the two sinusoids are $80 \mathrm{~Hz}$ and $180 \mathrm{~Hz}$. At $\mathrm{t}=25 \mathrm{~s}$ one switches to $70 \mathrm{~Hz}$ and $130 \mathrm{~Hz}$. At $\mathrm{t}=50 \mathrm{~s}$ one returns to $80 \mathrm{~Hz}$ and $180 \mathrm{~Hz}$ and at $\mathrm{t}=75$, one switches to $90 \mathrm{~Hz}$ and $190 \mathrm{~Hz}$. The corresponding evolution of the parameters is shown in Fig. 17, Table 6 
summarizes steady states global attenuation results for the case of two simultaneous sinusoidal disturbances with distinct frequencies.

\begin{tabular}{ccccccc}
\hline Frequency $(\mathrm{Hz})$ & $80 / 180$ & $90 / 190$ & $130 / 170$ & $80 / 110$ & $80 / 150$ & $90 / 120$ \\
\hline Model Go, 60 par.(dB) & 85.95 & 90.72 & 67.04 & 75.33 & 72.21 & 76.02 \\
\hline
\end{tabular}

Table 4: Steady state attenuation for two simultaneous sinusoidal disturbances with distinct frequencies.

\subsubsection{The case of 3 sinusoidal disturbances of distinct frequencies}

In this case, in order to assure both disturbance rejection and stability, the order of the Q filter has been further augmented. A value of $\mathrm{nQ}=79$ (80 parameters) has been chosen. Fig. 18 displays the time response of the residual error for the rejection of three sinusoidal disturbances located at 70, 135 and $200 \mathrm{~Hz}$. The system operates in open loop for the first 5 s. Fig. 19 shows the power spectral density in open loop and in closed loop. One notices the strong attenuation of the fundamentals and of the first harmonics. Table 5 gives the steady state attenuation for various combination of the frequencies of the three sinusoidal disturbances.

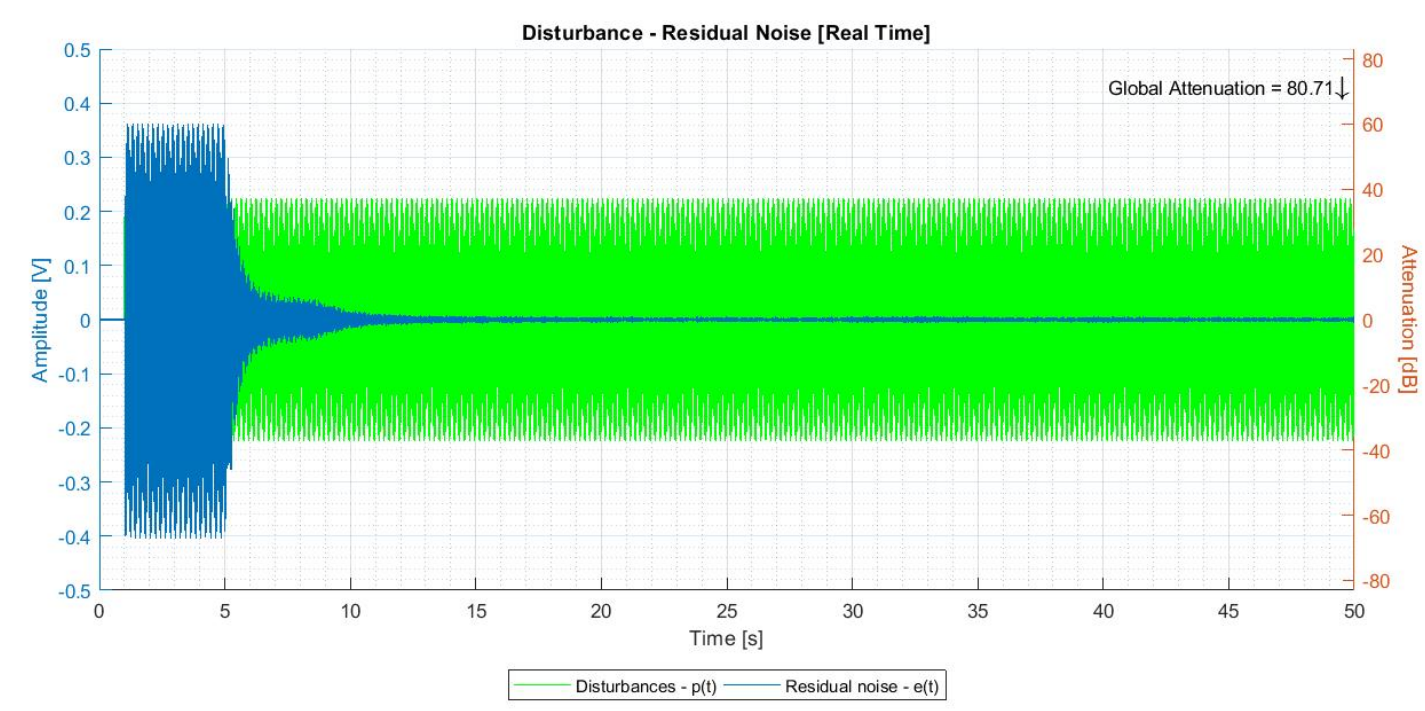

Figure 18: Evolution of the residual noise for three sinusodal disturbances (70/135/200 Hz - 80 parameters).

\subsubsection{The case of 4 sinusoidal disturbances of distinct frequencies}

In this case also a $\mathrm{Q}$ filter with 80 parameters have been used. The same experimental protocol as for the case of three sinusoidal disturbances has been used. It was observed that the convergence towards the steady state attenuation is slightly slower and that the constant trace algorithm provides better results than the constant gain algorithm. The results presented have been obtained with the constant trace algorithm. Fig. 20 shows the time response of the residual error for the rejection 


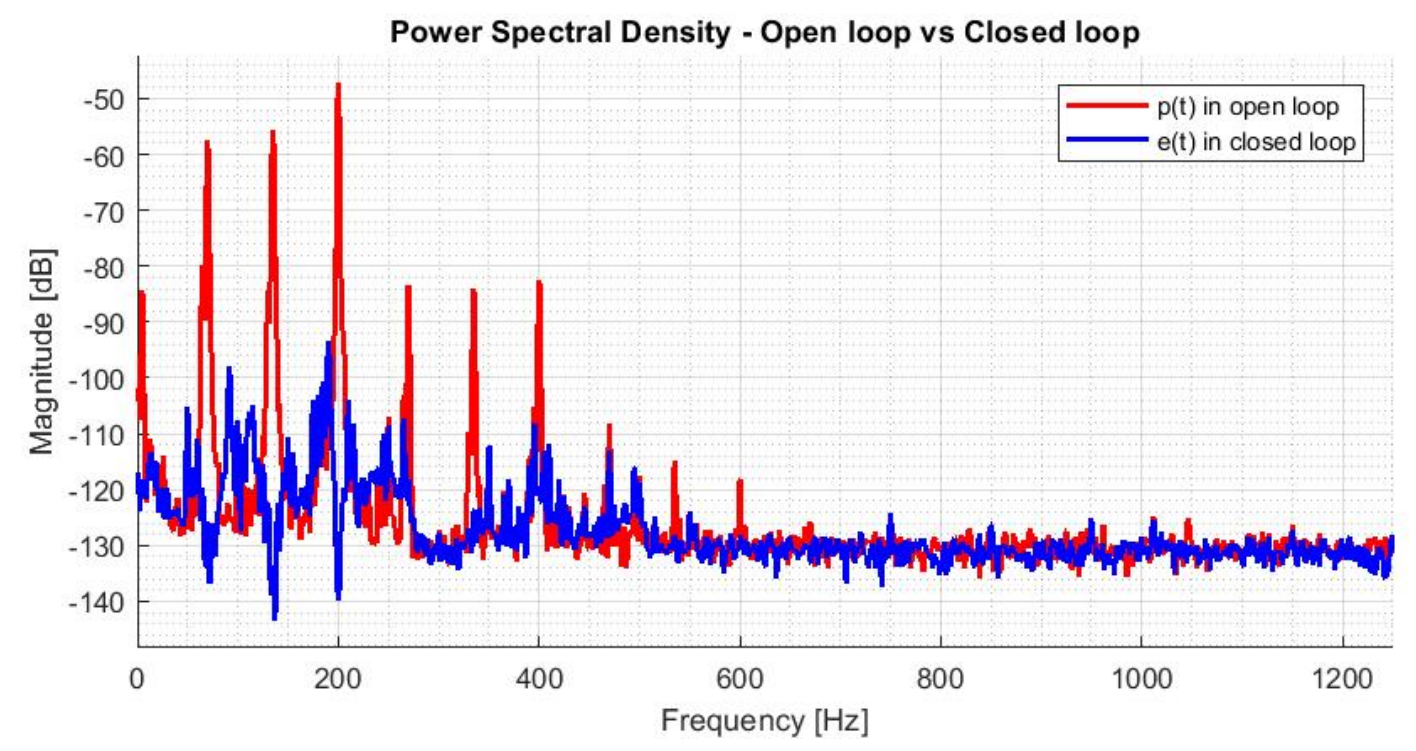

Figure 19: Power Spectral density of the residual noise in open loop and in closed loop for three sinusoidal disturbances $(70 / 135 / 200 \mathrm{~Hz}-80$ parameters).

\begin{tabular}{ccccc}
\hline Frequency $(\mathrm{Hz})$ & $70 / 95 / 120$ & $70 / 135 / 200$ & $70 / 145 / 220$ & $80 / 120 / 160$ \\
\hline Mod. Go, 80 par.(dB) & 66.69 & 80.71 & 73.91 & 69.33 \\
\hline Frequency $(\mathrm{Hz})$ & $90 / 115 / 140$ & $100 / 125 / 150$ & $140 / 160 / 180$ & $170 / 195 / 220$ \\
\hline Mod. Go, 80 par.(dB) & 66.19 & 64.81 & 60.58 & 79.73 \\
\hline
\end{tabular}

Table 5: Steady state attenuation for three simultaneous sinusoidal disturbances with distinct frequencies.

of four sinusoidal disturbances located at 70, 135 and $200 \mathrm{~Hz}$. Since the convergence is slightly slower, a time horizon of 100 s has been selected (instead of 50s for the previous disturbance configurations). Fig. 21 shows the power spectral density in open loop and in closed loop for this disturbance. One notices the strong attenuation of the fundamentals and of the first harmonics below $300 \mathrm{~Hz}$.

\begin{tabular}{ccccc}
\hline Frequency $(\mathrm{Hz})$ & $70 / 110$ & $70 / 115$ & $70 / 120$ & $90 / 130$ \\
& $150 / 190$ & $160 / 205$ & $170 / 220$ & $170 / 210$ \\
\hline Mod. Go, 80 par.(dB) & 66.69 & 80.71 & 73.91 & 69.33 \\
\hline
\end{tabular}

Table 6: Steady state attenuation for four simultaneous sinusoidal disturbances with distinct frequencies.

\subsubsection{Multiplicity of solutions}

Since one uses an overparametrized Q filter with respect to the complexity of the disturbance model, the solution for the asymptotic rejection of the disturbances is not unique and the values of the $\mathrm{Q}$ filter will depend on the initial conditions and previous characteristics of the disturbance. 


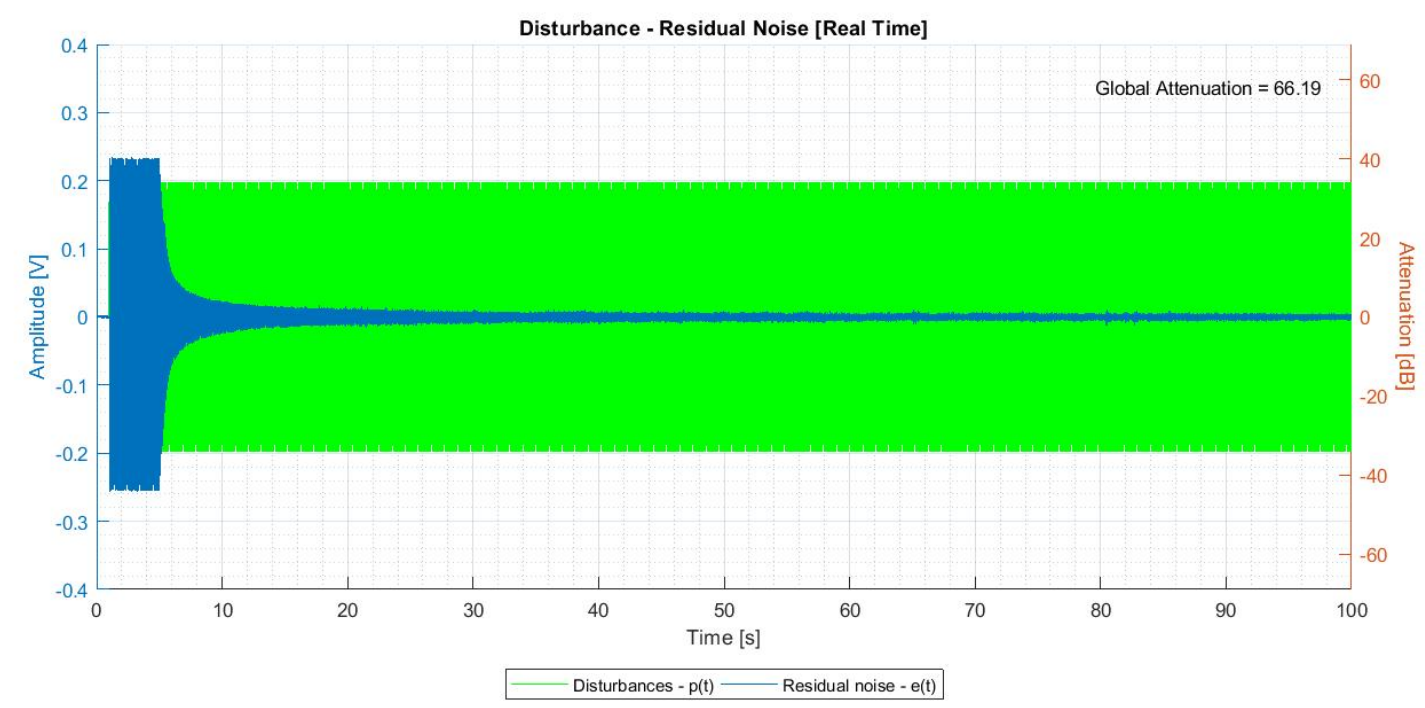

Figure 20: Evolution of the residual noise for four sinusoidal disturbances (70/120/170/220 Hz - 80 parameters).

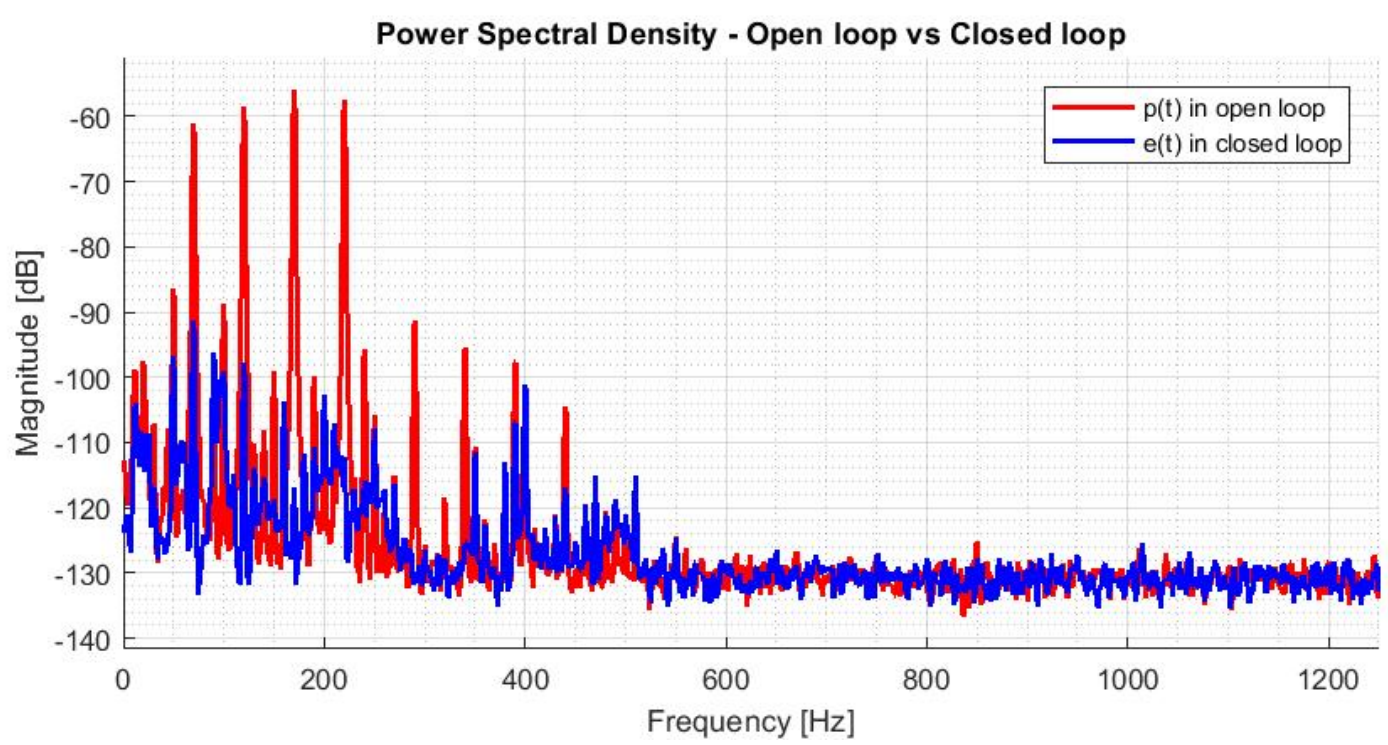

Figure 21: Power Spectral density of the residual noise in open loop and in closed loop for four sinusoidal disturbances (70/120/170/220 Hz - 80 parameters).

Not all of these solutions assure the stability of the system. Therefore once the parameters go beyond certain limits it is necessary to keep them within certain limits as indicated by theoretical analysis developed in this paper. For specific excitation protocols, this phenomenon is visible. Once this limit is reached, the algorithm is re-initialized at 0 for all the parameters (projection to the origin). Fig. 22 shows the evolution of the residual noise for a succession of step frequencies changes in an interference phenomenon around $160 \mathrm{~Hz}$ and $160.5 \mathrm{~Hz}$. A cycle of 4 steps changes 
in frequency of $120 \mathrm{~s}$ duration is repeated 10 times over an horizon of $1200 \mathrm{~s}$. The associated evolution of the parameters is shown in Fig. 23. One observes a drift in the parameters of the Q filter despite the fact the performances are repetitive. As soon as one of the parameters reaches the value 15 (which was set for illustrating the procedure), all the parameters are reinitialized at 0. This is clearly visible in Fig. 24 which is a zoom on the Fig. 22.

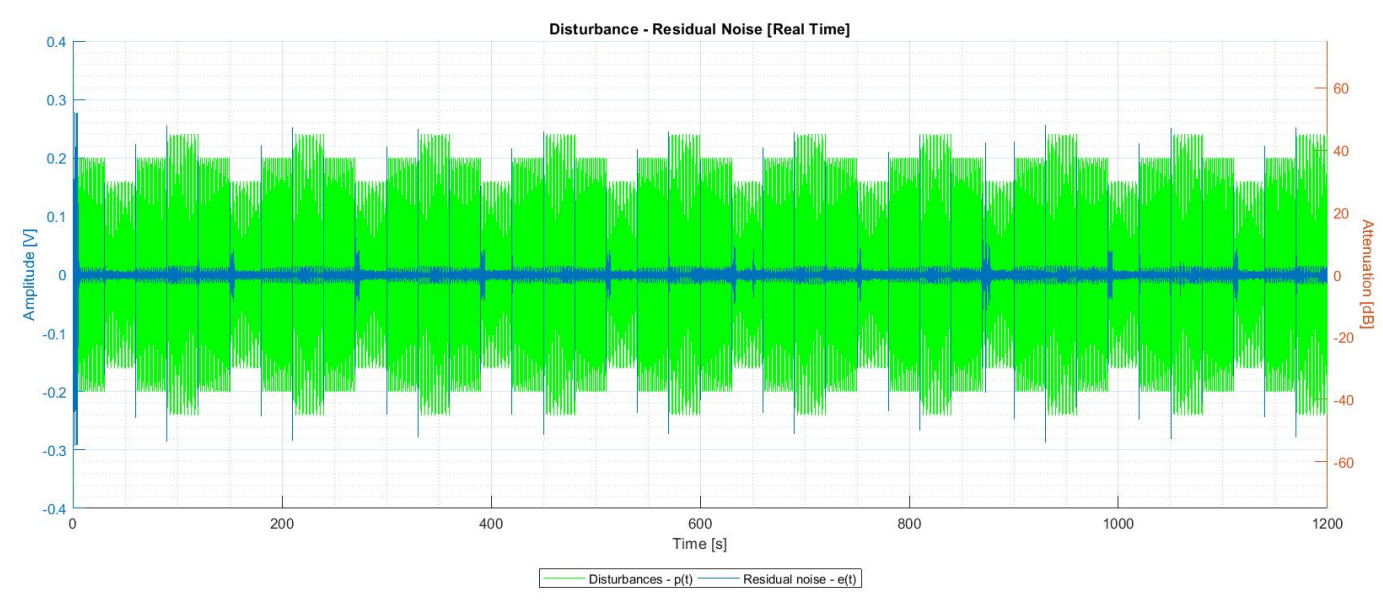

Figure 22: Evolution of the residual noise for step changes in the frequencies of an interference phenomenon over an horizon of 1200 s

\section{Conclusion}

The paper has shown that it is possible to handle large uncertainties on the model of the secondary (compensatory) path in adaptive feedback noise attenuation schemes using overparametrization of the compensation filter. However the feasible operation region depends upon a frequency condition related to the discrepancy between the design model and the real model and in addition a linear feedback design has to be done in order to satisfy a frequency condition for stability. Because of overparametrization, the standard parameter adaptation algorithm has to be completed with a projection procedure.

Current research is directed towards allowing a larger discrepancy between the design model and the real model.

Appendix A. Derivation of Eq. (19)

By combining Eqs. (5) and (6), one has $w(t)=-B_{0} u(t)+A_{0}\left[\frac{B}{A} u(t)+d(t)\right]=\left(-B_{0}+A_{0} \frac{B}{A}\right) u(t)+$ $A_{0} d(t)$, and by including Eq. (15) in the previous equation, one obtains $w(t)=\left(-B_{0}+A_{0} \frac{B}{A}\right)\left[-\frac{H_{R}}{S_{0}} Q w(t)\right]+$ $A_{0} d(t)$, which yields $\left[A S_{0}+H_{R} Q\left(A_{0} B-A B_{0}\right] w(t)=A_{0} A S d(t)\right.$, hence the result. 


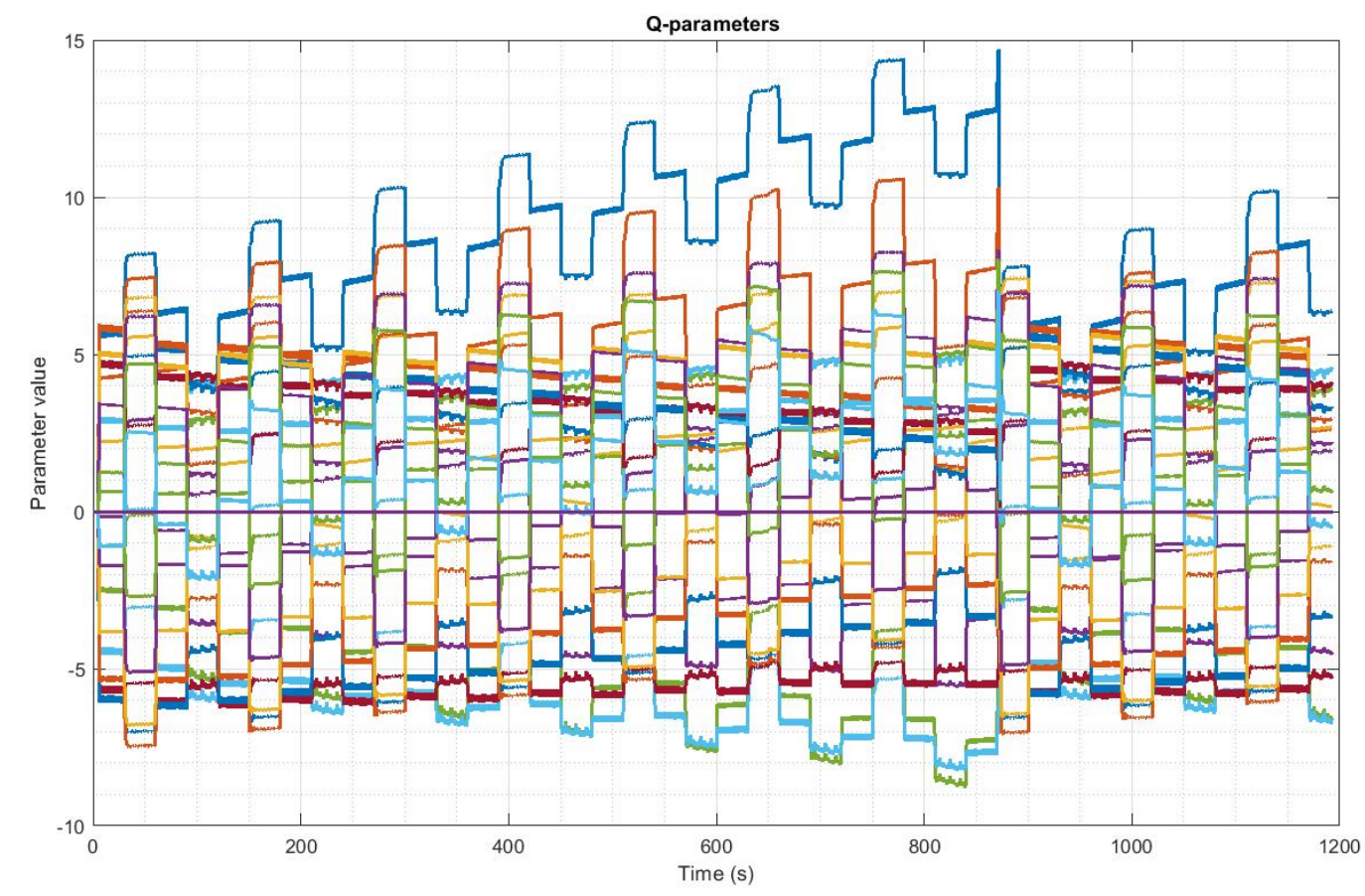

Figure 23: Evolution of the parameters for step changes in in the frequencies of an interference phenomenon over an horizon of 1200 s

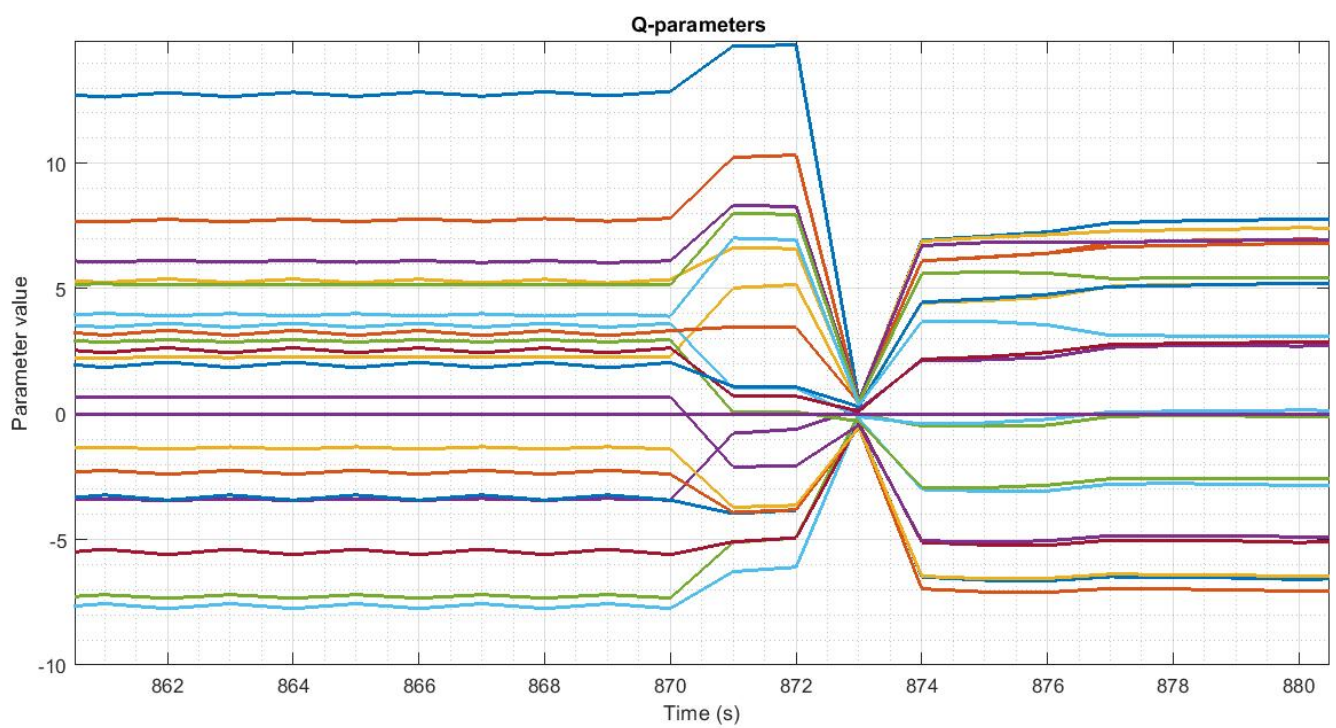

Figure 24: Zoom on the evolution of the parameters at resetting 
Appendix B. Derivation of Eq. (20)

By combining Eqs. (19) and (20) one has $u(t)=-\frac{H_{R}}{S_{0}} Q_{\frac{A S_{0} A_{0}}{A S_{0}+Q H_{R}\left(B A_{0}-B_{0} A\right)}} d(t)$, thus $y(t)=\frac{B}{A}-$ $\frac{H_{R}}{S_{0}} Q_{\frac{A S_{0} A_{0}}{A S_{0}+Q H_{R}\left(B A_{0}-B_{0} A\right)}} d(t)+d(t)$ which leads to the expression $y(t)=\frac{\left[-B H_{R} Q A_{0}+A S_{0}+H_{R} Q\left(A_{0} B-A B_{0}\right)\right]}{A S_{0}+H_{R} Q\left(A_{0} B-A B_{0}\right)} d(t)$ and one gets $y(t)=\frac{A S_{0}-H_{R} Q A B_{0}}{A S_{0}+H_{R} Q\left(A_{0} B-A B_{0}\right)} d(t)$, and the result is obtained.

\section{Appendix C. Derivation of conditions (22) and (23)}

According to the expression of the direct sensitivity function (20), the closed-loop is stable if and only if the polynomial $A S_{0}+H_{R} Q\left(A_{0} B-A B_{0}\right)$ has all its roots strictly inside the unit circle. This stability condition is equivalent to the stability condition of the closed loop shown in Fig. C.25]. and according to the small gain theorem ([9]) condition 2) is obtained. Now at

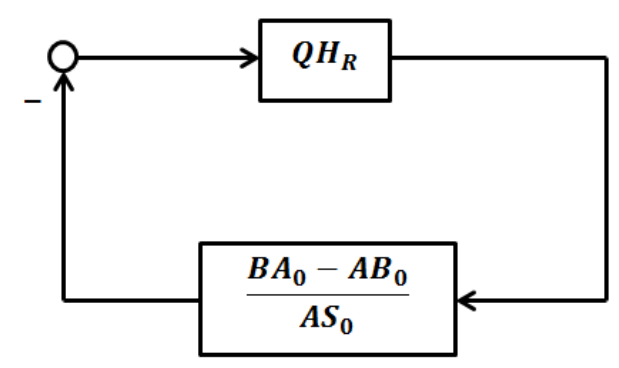

Figure C.25: Equivalent closed-loop

the frequencies $\omega_{j}$ of the disturbance, where $D\left(e^{-i \omega_{j}}\right)=0$, the Bezout equation (13), becomes $H_{R}\left(e^{-i \omega_{j}}\right) B_{0}\left(e^{-i \omega_{j}}\right) Q\left(e^{-i \omega_{j}}\right)=S_{0}\left(e^{-i \omega_{j}}\right)$, therefore $Q=\frac{S_{0}\left(e^{-i \omega_{j}}\right)}{H_{R}\left(e^{-i \omega_{j}}\right) B_{0}\left(e^{-i \omega_{j}}\right)}$ and condition 2) becomes

$$
|S|=\left|1-\frac{B\left(e^{-i \omega_{j}}\right) A_{0}\left(e^{-i \omega_{j}}\right)}{A\left(e^{-i \omega_{j}}\right) B_{0}\left(e^{-i \omega_{j}}\right)}\right|<1
$$

for all $\omega_{j}$. By using the results of [9] (p. 564) concerning the equivalence between the small gain theorem and the asymptotic hyperstability (passivity) theorem, one gets from Eq (C.73), p. 564 that the equivalent expression of the transfer function which should be positive real is given by $H=(1+S) /(1-S)$ which leads to the condition: $\frac{A\left(z^{-1}\right) B_{0}\left(z^{-1}\right)}{A\left(z^{-1}\right) B_{0}\left(z^{-1}\right)}-\frac{1}{2}$ is a strictly positive real transfer function for all $\omega_{j}$ and condition 1$)$ is proved.

\section{Appendix D. Stability analysis of the adaptive scheme}

The following theorem summarizes the stability analysis of the adaptive scheme

Theorem 1. Under the assumptions: 
- Plant $G$ is asymptotically stable.

- Disturbance d(t) has the form (4) and is bounded

- It exists $Q\left(q^{-1}\right) \in \mathscr{D}$ with $n_{D}-1 \leq n_{Q}<\infty$ satisfying (13) and assuring that the polynomial (18) has all its roots strictly inside the unit circle.

then one has:

$$
\lim _{t \rightarrow \infty} v(t+1)=0
$$

and if in addition:

$$
\max _{\hat{\Theta} \in \mathscr{D}}\left(\|\hat{Q}-Q\|_{1}\right)\left\|\frac{H_{R}\left(B A_{0}-B_{0} A\right)}{A S_{0}+Q H_{R}\left(B A_{0}-B_{0} A\right)}\right\|_{1}<1
$$

one has:

$$
w(t), u(t), y(t) \text { are bounded }
$$

and:

$$
\lim _{t \rightarrow \infty} v^{o}(t+1)=\lim _{t \rightarrow \infty} y(t+1)=0
$$

Remark : For $G=G_{0}$, condition (D.2) is automatically satisfied. Similar situation occurs for the case $n_{Q}=n_{D}-1$ if $(18)$ is satisfied, since the persistence of excitation drives $\tilde{Q}(t)=\hat{Q}(t)-Q$ to zero.

Proof: The equation of the a posteriori adaptation error (32) has the form considered in Theorem 3.2 from [9] (pp 104-105) and since $H\left(q^{-1}\right)=1$, one immediately concludes that (D.1) holds. To go further one has to show that $\mathbf{w}(t)$ and respectively $w(t)$ are bounded $(\mathbf{w}(t)$ contains delayed and filtered signals generated by $w(t))$.

Set $\Gamma=A S_{0}$ and $\bar{\Delta}=H_{R}\left(B A_{0}-A B_{0}\right)$. In an adaptive context using (19), one can write:

$$
[\Gamma+\bar{\Delta} \hat{Q}(t)] w(t)=\left(\Gamma A_{o}\right) d(t)
$$

or alternatively:

$$
[\Gamma+\bar{\Delta} \hat{Q}(t) \pm \bar{\Delta} Q] w(t)=\left(\Gamma A_{o}\right) d(t)
$$

Set $N=\Gamma A_{o}$, and $\tilde{Q}(t)=\hat{Q}(t)-Q$ and $(\overline{D .6})$ can be written as:

$$
w(t)=\frac{N}{\Gamma+\bar{\Delta} Q} d(t)-\tilde{Q}(t) \frac{\bar{\Delta}}{\Gamma+\bar{\Delta} Q} w(t)
$$

which corresponds to a feedback system with an external bounded excitation whose output is $w(t)$, shown in Fig. D.26. The input to the equivalent feedback system $\bar{d}=\frac{N}{\Gamma+\bar{\Delta} Q} d(t)$ has the property:

$$
\|\overline{d(t)}\|_{\infty} \leq\left\|\frac{N}{\Gamma+\bar{\Delta} Q}\right\|_{1} d_{m}(t)
$$


where $d_{m}(t)=\sup |d(t)|=\|d(t)\|_{\infty}$ and $\left\|\frac{N}{\Gamma+\bar{\Delta} \bar{Q}}\right\|_{1}$ is finite. The output of the feedback path $x(t)$ is given by

$$
x(t)=\frac{\bar{\Delta} \tilde{Q}}{\Gamma+\bar{\Delta} Q} w(t)
$$

and therefore:

$$
\|w(t)\|_{\infty} \leq\|\bar{d}(t)\|_{\infty}+\|\tilde{Q}\|_{1} \mid \frac{\bar{\Delta}}{\Gamma+\bar{\Delta} Q}\left\|_{1}\right\| w(t) \|_{\infty}
$$

Applying small gain arguments [22] and taking into account that the equivalent feedforward path has unitary gain one concludes that condition (D.2) assures the boundedness of w(t). From the definition of $\mathbf{r}(t)$ given in (29), one concludes also that $\mathbf{w}(t)$ is bounded and taking in account (34) one concludes that (D.4) is true. Since $y(t)$ is bounded in finite time (and goes to zero), $w(t)$ is bounded and $Q$ is bounded, one concludes that $u(t)$ is also bounded.

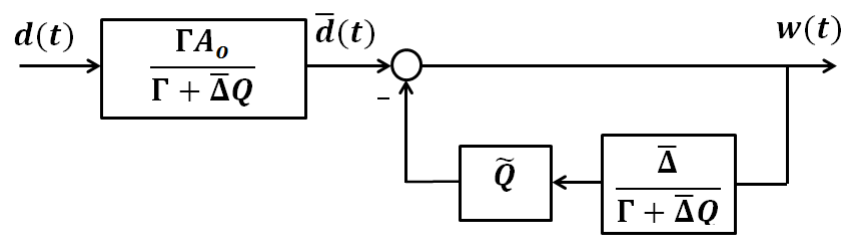

Figure D.26: Generation of $w(t)$.

\section{References}

[1] B. Francis, W. Wonham, The internal model principle of control theory, Automatica 12 (5) (1976) 457 - 465.

[2] I. Landau, A. Constantinescu, D. Rey, Adaptive narrow band disturbance rejection applied to an active suspension - an internal model principle approach, Automatica 41 (4) (2005) 563-574.

[3] F. Ben Amara, P. Kabamba, A. Ulsoy, Adaptive sinusoidal disturbance rejection in linear discrete-time systems - Part I: Theory, Journal of Dynamic Systems Measurement and Control 121 (1999) 648-654.

[4] I. Landau, R. Melendez, L.Dugard, G. Buche, Robust and adaptive noise attenuation in ducts, IEEE Trans. on Control Systems Technology 37 (2) (2019) 872-879.

[5] I. D. Landau, A. C. Silva, T.-B. Airimitoaie, G. Buche, M. Noé, Benchmark on adaptive regulation - rejection of unknown/time-varying multiple narrow band disturbances, European Journal of Control 19 (4) (2013) 237 252.

[6] R. Melendez, I. Landau, L. Dugard, G. Buche, Data driven design of tonal noise feedback cancellers, in: Proceedings of the 20th IFAC World Congress, Toulouse, France, 2017, pp. 916-921.

[7] I. Landau, R. Melendez, T. Airimitoaie, L.Dugard, Beyond the delay barrier in adaptive feedforward active noise control using youla-kucera parametrization, Journal of Sound and Vibration 455 (9) (2019) 339-358.

[8] Q. Hang, S. Chen, M. Huang, Z. Guo, Adaptive active noise suppression using multiple model switching strategy, Shock and Vibration 2017, ID 7289076.

[9] I. D. Landau, R. Lozano, M. M’Saad, A. Karimi, Adaptive control, 2nd Edition, Springer, London, 2011.

[10] K. Fujii, J. Ohga, Method to update the coefficients of the secondary path filter under active noise control, Journal of Signal Processing 81 (6) (2001) 381-387.

[11] H. Zheng, D. Yang, X. Xie, An adaptive algorithm for active vibration control of parametervarying systems with a new online secondary path estimation method, IEEE Signal Processing Letters 27 (2020) 705-709. 
[12] G. Jin, T. Yang, Y. Xiao, A simultaneous equation method based online secondary path modeling algorithm for active noise control, Journal of Sound and Vibration 303 (3-5) (2007) 455-474.

[13] W. Niu, C. Zou, B. Li, W. Wang, Adaptive vibration suppression of time-varying structures with enhanced fxlms algorithm, Mechanical Systems and Signal Processing 118 (2019) 93-107.

[14] S. Valentinotti, Adaptive rejection of unstable disturbances: Application to a fed-batch fermentation, Thèse de doctorat, École Polytechnique Fédérale de Lausanne (April 2001).

[15] S.Jafari, P. Ioannou, B. Fitzpatrick, Y. Wang, Robustness and performance of adaptive suppression of unknown periodic disturbances, IEEE Transactions on Automatic Control 60 (8) (2015) 2166-2171.

[16] C. Kinney, H. Fang, R. de Callafon, M. Alma, Robust estimation and automatic controller tuning in vibration control of time varying harmonic disturbances, in: Proceedings of the 18th IFAC World Congress, Milano, Italy, 2011, pp. 5401-5406.

[17] B. Anderson, From Youla-Kučera to identification, adaptive and nonlinear control, Automatica 34 (12) (1998) 1485 - 1506.

[18] P. Mullhaupt, D. Bonvin, Asymptotic rejection of nonvanishing disturbances despite plant-model mismatch, Int. J. of Adapt. Control Signal Process 26(12) (2012) 1090-1110.

[19] B.Vau, I. Landau, Youla-kucera adaptive feedback disturbance rejection in the presence of plant uncertainties, in: Proceedings of the 58th IEEE Conf. on Decision and Control, Nice, France, 2019, pp. 102-107.

[20] I. D. Landau, T.-B. Airimitoaie, A. Castellanos Silva, A. Constantinescu, Adaptive and Robust Active Vibration Control-Methodology and Tests, Advances in Industrial Control, Springer, London, 2017.

[21] S. Haykin, Adaptive Filter Theory, Prentice Hall, Englewood Cliffs N.J., 1996.

[22] C. Desoer, M. Vidyasagar, Feedback Systems: Input-Output properties, Academic Press, Cambridge, 1975. 